\title{
Zivilrechtliche Haftung für den Einsatz von Robotern - Zuweisung von Automatisierungs- und Autonomierisiken
}

\author{
Prof. Dr. iur. Dipl.-Biol. Herbert Zech, Universität Basel
}

„Wir sind auf alles programmiert und was du willst wird ausgeführt.

Wir sind die Roboter.“1

\section{Einleitung}

Die Robotertechnologie vollzieht gerade entscheidende Entwicklungsschritte für eine weit verbreitete Anwendung. Sie ist dabei, das unternehmerische Umfeld zu verlassen und in den privaten Bereich vorzudringen. Wesentliches Merkmal dieser Entwicklung ist die zunehmende Komplexität der Steuerung, die eine immer weitergehende Automatisierung erlaubt. Roboter werden auch in den öffentlichen Raum vordringen bzw. am allgemeinen Verkehr teilnehmen, wofür wichtigstes Beispiel die Teilnahme selbststeuernder Fahrzeuge am Straßenverkehr darstellt. Als noch weitergehende Entwicklung werden schließlich auch autonome Verhaltensweisen erzielbar sein, insbesondere durch die Fortschritte im Bereich des deep learning.

Diese Entwicklung lässt neuartige Risiken beim Einsatz von Robotern entstehen. Dadurch stellt sich auch die Frage nach dem passenden zivilrechtlichen Haftungsregime, mit dem der grundsätzlich erwünschte Einsatz von Robotern ermöglicht, damit verbundene Risiken aber gerecht und effizient verteilt werden. Der Beitrag beschäftigt sich mit der Haftung der Hersteller und der Anwender von Robotern nach geltendem Recht und zeigt mögliche rechtliche Gestaltungsoptionen auf. ${ }^{2}$

1 Kraftwerk, Die Roboter, in: Die Mensch-Maschine (Album), 1978, Songtext von Ralf Hütter.

2 Zwischen Abgabe und Drucklegung sind neben den zitierten weitere einschlägige Veröffentlichungen erschienen, die nicht mehr berücksichtigt werden konnten: $G$. Borges, Haftung für selbstfahrende Autos - Warum eine Kausalhaftung für selbstfahrende Autos gesetzlich geregelt werden sollte, CR 2016, S. 272 ff.; F. Freytag, Sicherheitsrechtliche Aspekte der Robotik, S\&R 2016, S. 111 ff.; S. Gless/R. Janal, 
Die Untersuchung konzentriert sich auf automatisierte mobile Maschinen, deren Verbreitung bereits im Gange ist, und autonome Maschinen, die zmindest in den nächsten Jahrzehnten eingeführt werden dürften. Die mögliche Entstehnung von Selbstbewusstsein und dessen rechtliche Konsequenzen bleiben weitestgehend außer Betracht. Bei den rechtlichen Aspekten werden sicherheits- und strafrechtliche Aspekte nur gelegentlich angesprochen. Zentrales Thema ist die zivilrechtliche Haftung, wiederum fokussiert auf Hersteller und Anwender von Robotern.

In einem ersten Teil (A), der sich mit den faktischen Grundlagen beschäftigt, werden die besonderen Risiken der Robotertechnologie herausgearbeitet, die für das Haftungsrecht von Interesse sind. Dabei soll vor allem gezeigt werden, welche neuartigen Risiken die technische Entwicklung mit sich bringt. Der zweite Teil (B) beschäftigt sich mit den rechtlichen Grundlagen. Er veranschaulicht, dass es sich bei der Roboterhaftung um einen speziellen Fall der Haftung für technische Produkte handelt. Er stellt die möglichen Verantwortlichen dar und zeichnet die Strukturen der Haftung für pflichtwidriges und pflichtgemäßes Verhalten im Umgang mit technischen Produkten nach.

Mit der Haftung nach geltendem Recht für hoch-automatisierte (C) und für autonome (D) Roboter beschäftigen sich die beiden folgenden Teile. Dabei wird jeweils zunächst die Herstellerseite, dann die Anwenderseite beleuchtet. In einem letzten Teil (E) soll erörtert werden, welche neuen Haftungsregelungen für Robotertechnologie eingeführt werden könnten. Dabei geht es vor allem um Gefährdungshaftungsregelungen, die die Einführung und Verbreitung erwünschter, aber riskanter Technik erleichtern könnten.

Hochautomatisiertes und autonomes Autofahren - Risiko und rechtliche Verantwortung, JR 2016, S. 561 ff.; V. Kluge/A.-K. Müller, Autonome Systeme - Überlegungen zur Forderung nach einer ,Roboterhaftung“, in: J. Taeger (Hrsg.), Smart World - Smart Law?, Oldenburg 2016, S. 989 ff.; C. Lötscher, Wenn das Auto den Laster nicht sieht - Verschiebung zivilrechtlicher Verantwortlichkeit aufgrund intelligenter Algorithmen?, Jusletter IT (im Erscheinen); J. Reichwald/D. Pfisterer, Autonomie und Intelligenz im Internet der Dinge Möglichkeiten und Grenzen autonomer Handlungen, CR 2016, S. 208 ff.; G. Spindler, Digitale Wirtschaft - analoges Recht: Braucht das BGB ein Update?, JZ 2016, S. 805, 815 f. 


\section{A. Besondere Risiken von Robotern}

Als Robotics bzw. Robotertechnologie soll derjenige Bereich der Technik verstanden werden, der sich mit IT-gesteuerten Maschinen (Verknüpfung von Sensoren, Computersteuerung und Aktuatoren) ${ }^{3}$ bzw. künstlichen Agenten, die sich in der realen Welt bewegen, ${ }^{4}$ beschäftigt. Es geht also um die Anwendung (daher Technik) von computergesteuerten (Informationstechnik, IT) Geräten mit beweglichen Teilen (Maschinen) $)^{5}$. Dieser Bereich der Technik ist beinahe ebenso alt wie die IT selbst, macht aber derzeit entscheidende Fortschritte. Daraus ergeben sich Besonderheiten gegenüber anderen Technikbereichen, die auch neuartige Risiken zur Folge haben.

\section{Entwicklung der Robotertechnologie (Robotics)}

Nach Dominik Bösl kann man vier Entwicklungsschritte in der Robotertechnologie unterscheiden, beginnend mit der Einführung automatisierter Steuerungen, die bereits vier Jahrzehnte zurückliegt, über komplexere

3 G. A. Bekey, Autonomous Robots, London 2005, S. 2: “[...] we define a robot as a machine that senses, thinks, and acts. Thus, a robot must have sensors, processing ability that emulates some aspects of cognition, and actuators." Vgl. Th. Christaller u.a., Robotik: Perspektiven für menschliches Handeln in der zukünftigen Gesellschaft, Berlin/Heidelberg 2001, S. 18 ff.; M. F. Lohmann, Roboter und Recht, AJP 2014, S. 595, 596.

4 St. Russell/P. Norvig, Artificial Intelligence - A Modern Approach, 3. Aufl., Harlow 2016, S. 34: "An agent is anything that can be viewed as perceiving its environment through sensors and acting upon that environment through actuators. [...] A human agent has eyes, ears and other organs for sensors and hands, legs, vocal tract, and so on for actuators. A robotic agent might have cameras and infrared range finders for sensors and various motors for actuators. A software agent receives keystrokes, file contents, and network packets as sensory inputs and acts on the environment by displaying on the screen, writing files, and sending network packets."

5 Vgl. Definition von Maschine nach Art. 2 Abs. 2 lit. a Richtlinie 2006/42/EG des Europäischen Parlaments und des Rates vom 17. Mai 2006 über Maschinen und zur Änderung der Richtlinie 95/16/EG (Neufassung), insbesondere die Grunddefinition im ersten Spiegelstrich: ,eine mit einem anderen Antriebssystem als der unmittelbar eingesetzten menschlichen oder tierischen Kraft ausgestattete oder dafür vorgesehene Gesamtheit miteinander verbundener Teile oder Vorrichtungen, von denen mindestens eines bzw. eine beweglich ist und die für eine bestimmte Anwendung zusammengefügt sind“". 
Steuerungen mit Sensoren, die eine unmittelbare Interaktion mit Menschen erlauben, bis zu mobilen und zuletzt autonomen Robotern. ${ }^{6}$ Die beiden letzten Phasen, frei sich in der Umwelt bewegende und autonome Roboter, liegen noch in der näheren bzw. ferneren Zukunft. Selbststeuernde Fahrzeuge werden der wohl wichtigste Anwendungsfall mobiler Roboter sein (aber auch Drohnen oder Serviceroboter), dessen Einführung in nicht allzu ferner Zukunft zu erwarten ist. Autonome Roboter (zum Begriff weiter unten) werden vermutlich noch etwas länger auf sich warten lassen (Einführung in den nächsten zwei Jahrzehnten). ${ }^{7}$ Für die weitere Untersuchung soll zwischen drei Technologieschritten unterschieden werden: der klassischen Robotertechnologie, die bereits angewendet wird, hoch- und vollautomatisierten mobilen Robotern, deren Verbreitung unmittelbar bevorsteht, und autonome Roboter, deren Entwicklung und Verbreitung erst im nächsten und übernächsten Jahrzehnt zu erwarten ist.

\section{Was macht Robotics zu einer besonderen Technologie?}

Die Verknüpfung von Sensoren, informationstechnischen Systemen und Aktuatoren ist nichts Neues. Die stetige Fortentwicklung der Robotertechnologie in den letzten Jahrzehnten, insbesondere aber die technischen Durchbrüche innerhalb der letzten Dekade, führen zu mehreren Besonderheiten, die Roboter von herkömmlichen Maschinen unterscheiden. Gill Pratt nennt als entscheidende Entwicklungen, die den Einsatz von Robotern praktikabel und nützlich machen (,technical drivers“): exponentielles Wachstum der Computerleistung, Verbesserung der Geräte für die Herstellung (insbesondere 3D-Druck), Verbesserungen bei der Speicherung elektrischer Energie, Verbesserung der Energieeffizienz elektronischer Geräte, exponentielles Wachstum von Größe und Leistung des Internet, exponentielles Wachstum der weltweiten Datenspeicherung und exponentielles

6 Nach Ph. Krohn, Streichel mich, sonst ersetz ich dich, FAZ, 19.2.2016, S. 16.

7 K. Mainzer, Computer, künstliche Intelligenz und Theory of Mind: Modelle des Menschlichen?, in: H. Förstl (Hrsg.), Theory of Mind - Neurobiologie und Psychologie sozialen Verhaltens, 2. Aufl., Berlin/Heidelberg 2012, Kap. 12, S. 145: „Es wird angenommen, dass sich Roboter bereits in den kommenden beiden Jahrzehnten komplexen und unstrukturierten Umgebungen anpassen können und Menschen bei der Erledigung von Alltagsaufgaben assistieren. Ihre Autonomie wirft eine Reihe von Sicherheitsfragen auf.“ 
Wachstum der weltweiten Gesamt-Computerleistung. ${ }^{8}$ Durch diese Entwicklungen entstehen Besonderheiten, die auch aus rechtlicher Sicht relevant sind.

\section{Klassische Robotics: Komplexität und Kopplung von Software und Hardware}

a) Komplexität von IT (Hardware und Software)

Informationstechnische Systeme zeichnen sich allgemein durch eine besondere Komplexität aus, die durch die fortschreitende Miniaturisierung immer weiter zunimmt. ${ }^{9}$ Die Menge an Information, die in einem bestimmten Volumen gespeichert werden kann, wächst z.B. exponentiell (Mooresches Gesetz). ${ }^{10}$

Höhere Komplexität führt grundsätzlich dazu, dass das Verhalten eines Systems schwerer vorhersagbar wird. In der Alltagserfahrung spiegelt sich dies in dem Umstand, dass Fehlfunktionen bei einem Computer (z.B. die plötzliche Beendigung laufender Programme) sehr viel häufiger vorkommen als bei einfachen elektronischen Geräten. Allerdings gibt es auch Strategien, mit denen das Verhalten komplexer Systeme stabilisiert werden kann, wie durch entsprechende Softwarearchitekturen. Auch Lebewesen stellen komplexe Systeme dar, die jedoch erstaunlich zuverlässig „funktionieren“.

Komplexität von IT ist kein neuartiges Risiko, sondern seit den 1970ern und 1980ern bekannt, durch Fortschritte der Informatik nimmt das Risiko offenbar eher ab.

8 G. A. Pratt, Is a Cambrian Explosion Coming for Robotics?, JEP 29, 2015, S. 51 (53 ff.).

9 E. Brynjolfsson/A. McAfee, The Second Machine Age, New York/London 2014, S. 8, gehen davon aus, dass sich die Entwicklung momentan an einem Punkt befindet der mit dem Stand der Entwicklung von Energiemaschinen bei Einführung der Watt'schen Dampfmaschine vergleichbar ist, d.h. eine schon länger andauernde technische Entwicklung erreicht den „Wendepunkt“ an einer „hockey stick-curve“. Dazu Frese, Recht im zweiten Maschinenzeitalter, NJW 2015, 2090, 2091.

10 Brynjolfsson/McAffe (Fn. 9), S. 39 ff. 
b) Kopplung von Software und Hardware

Was Roboter gegenüber anderen informationsverarbeitenden Systemen auszeichnet, ist die Verbindung von Softwaresteuerung und Elementen, die in der "realen“ Welt Kräfte entfalten können (Aktuatoren). ${ }^{11}$ Diese Kopplung erzeugt besondere Risiken, da Fehlfunktionen der Software unmittelbar mechanische Gefahren auslösen können. Zwar können in der modernen Welt auch reine Softwarenetze Gefahren bergen, doch führen diese wesentlich seltener zu Schäden an Leib und Leben als mechanische Gefahren. Die Diskussion um selbstfahrende Fahrzeuge führt dies deutlich vor Augen (Schäden durch Fehlsteuerung eines medizinischen Bestrahlungsgerätes, nämlich des „Therac-25“, in den 80er-Jahren wurde dagegen eher in Fachkreisen diskutiert ${ }^{12}$ ).

\section{Aktuelle Entwicklung: Zunehmende Automatisierung, Mobilität und Vernetzung}

a) Zunehmende Automatisierung (Hoch- und Voll-Automatisierung)

Auch die Automatisierung, d.h. die selbsttätige (ohne Hilfe von Menschen) Steuerung von Abläufen, durch Computer gehört zum Üblichen, das sich bei jedem Industrieroboter finden lässt. ${ }^{13}$ Die Automatisierung ändert nichts daran, dass das Verhalten des Roboters zumindest im regulären, d.h. nicht fehlerhaften, Betrieb vorhersagen lässt. Zwar birgt die automatisierte Steuerung von Maschinen besondere Risiken, die teilweise mit der Komplexität und damit der Fehleranfälligkeit der Steuerung durch Computer zu tun haben, teilweise mit der Automatisierung selbst (selbsttätige Bewegungsabläufe, Kraftentfaltung etc.). Diese Risiken sind aber von komplexen Maschinen generell bekannt.

11 Vgl. Bekey (Fn. 3), S. 2.

12 Dazu N. G. Leveson/C. S. Turner, An Investigation of the Therac-25 Accidents, Computer (IEEE Computer Society) 1993, S. 18 ff.; S. Baase, A Gift of Fire - Social, Legal, and Ethical Issues for Computing and the Internet, 3. Aufl., Upper Saddle River, NJ 2008, S. 425 ff.

13 Eine automatische Steuerung kann auch direkt mechanisch erfolgen, wie z.B. bei dem von James Watt erfundenen Fliehkraftregler für Dampfmaschinen. Roboter zeichnen sich jedoch durch eine komplexe informationstechnische Steuerung aus. 
Das eigentlich Neue besteht in der exponentiellen Zunahme der Leistungsfähigkeit, welche die steuernden Computer besitzen. Dies ist auf die zunehmende Miniaturisierung (Komplexität) und Energieeffizienz von Computern zurückzuführen.

Durch die höhere Leistungsfähigkeit wird ein immer höherer Grad an Automatisierung geschaffen, d.h. auch komplizierte Steuerungsvorgänge (wie etwa das Steuern eines Autos) können ohne Eingreifen des Menschen erfolgen. Dabei kann man verschiedene Automatisierungsgrade unterscheiden, die ein menschliches Eingreifen in unterschiedlichem Maße verzichtbar machen: ${ }^{14}$ Teilautomatisierung, Hochautomatisierung und Vollautomatisierung. Bei der Vollautomatisierung ist außer dem Einsatz der Maschine als solchem kein Eingreifen des Nutzers mehr erforderlich.

\section{b) Mobilität}

Durch bessere Energieversorgung (Speicherung) und -effizienz werden Roboter beweglich. Dies spielt für das Risiko, das mit dem Einsatz von Robotern verbunden ist, eine große Rolle. Roboter, die - wie etwa klassische Industrieroboter - ortsfest installiert sind, haben nur einen begrenzten Aktionsradius. Bewegliche Roboter, die in abgeschlossenen Räumen eingesetzt werden, stellen zumindest ein geringeres Risiko für die Allgemeinheit dar als Roboter, die sich im öffentlichen Raum bewegen.

\section{c) Vernetzung}

Ein weiterer wichtiger Aspekt der aktuellen Robotertechnik, der z.B. auch bei selbststeuernden Fahrzeugen eine große Rolle spielen wird, ist die zunehmende Vernetzung einzelner Roboter. Ein informationsverarbeitendes System lässt sich immer schwerer isoliert betrachten, sondern ist mit zahl-

$14 \mathrm{Zu}$ der Unterscheidung bei selbstfahrenden Fahrzeugen T. M. Gasser u.a., Rechtsfolgen zunehmender Fahrzeugautomatisierung, Bergisch Gladbach 2012, S. 8 f;; M. F. Lohmann, Automatisierte Fahrzeuge im Lichte des Schweizer Zulassungsund Haftungsrechts, Baden-Baden 2016, S. 52 ff.; J. M. Beer/A. D. Fisk/ W. A. Rogers, Toward a Framework for Levels of Robot Autonomy in Human-Robot Interaction, Journal of Human-Robot Interaction 3 (2014), S. 74 (78), unterscheiden zehn ,levels of automation". 
reichen anderen Systemen vernetzt, so dass ebenso gut auch die Umgebung als Teil der Maschine betrachtet werden kann. Klaus Mainzer spricht von der „Infrastrukturrobotik“. ${ }^{15}$ Dies hat nicht nur Auswirkungen auf das Risiko beim Einsatz von Robotern, sondern kann auch die Zurechnung von Risiken erschweren. Kommt es z.B. durch fehlerhafte Daten anderer Roboter zu einer Fehlfunktion, lässt sich der Verursachungszusammenhang unter Umständen nur schwer aufklären.

\section{Zukünftige Entwicklung: Autonome Roboter}

In der aktuellen Diskussion über künstliche Intelligenz geht es vor allem um autonomes Verhalten. Dies bedeutet, dass ein informationstechnisches System sein Verhalten auch ohne unmittelbaren Anlass von außen verändern kann. ${ }^{16}$ Autonome Systeme verändern also die ihrem Verhalten zu-

15 Persönlicher Vortrag am 5.10.2015, Universität Passau.

16 L. Floridi/J. W. Sanders, On the Morality of Artificial Agents, Minds and Machines 14, 2004, S. 349 (357) "autonomy means that the agent is able to change state without direct response to interaction: it can perform internal transitions to change its state. So an agent must have at least two states. This property imbues an agent with a certain degree of complexity and independence from its environment." (Floridi/Sanders charakterisieren intelligente Agenten durch interactivity, autonomy und adaptability); Bekey (Fn. 3), S. 1 f.: „Autonomy refers to systems capable of operating in the real-world environment without any form of external control for extended periods of time.”; Russell/Norvig (Fn. 4), S. 40: „To the extent that an agent relies on the prior knowledge of its designer rather than on its own percepts, we say that the agent lacks autonomy." Überblick über verschiedene Definitionen bei Beer/Fisk/Rogers (Fn. 14), S. 74 (76). Vgl. S. Beck, Roboter, Cyborgs und das Recht - von der Fiktion zur Realität, in: T. Spranger (Hrsg.), Aktuelle Herausforderungen der Life Sciences, Berlin 2010, S. 101; dies., Grundlegende Fragen zum rechtlichen Umgang mit der Robotik, JR 2009, S. 225 (226); M. F. Lohmann, Von vermenschlichten Maschinen und maschinisierten Menschen, in: Brändli u.a. (Hrsg.), Mensch und Maschine - Symbiose oder Parasitismus?, Bern 2014, S. 125 (130 ff.); C. D. Müller-Hengstenberg/St. Kirn, Intelligente (Software-)Agenten: Eine neue Herausforderung unseres Rechtssystems - Rechtliche Konsequenzen der „Verselbständigung“ technischer Systeme, MMR 2014, S. 225 (228f.) und MMR 2014, S. 307 (309); J.-Ph. Günther, Roboter und rechtliche Verantwortung: Eine Untersuchung der Benutzer- und Herstellerhaftung, 2016, S. $29 \mathrm{ff}$. Ähnlich „embodied robotics“, vgl. J.-Ph. Günther, Embodied Robots Zeit für eine rechtliche Neubewertung, in: M.-Ch. Gruber/J. Bung/S. Ziemann (Hrsg.), Autonome Automaten - Künstliche Körper und artifizielle Agenten in der technisierten Gesellschaft, 2. Aufl, Berlin 2015, S. 155 (157 ff).; Mainzer, (Fn. 7), 
grundeliegenden Regeln (Algorithmen) selbständig. Ein solches selbstlernendes Verhalten wird durch die Leistungsfähigkeit moderner informationsverarbeitender Systeme ermöglicht. Eine besondere Rolle spielt dabei, das sogenannte deep learning ${ }^{17}$, Algorithmen, die große Datenmengen verarbeiten und daraus Verhaltensänderungen generieren können. Deep learning hat echte Autonomie Wirklichkeit werden lassen und liegt auch spektakulären Erfolgen künstlicher Intelligenz in den letzten zehn Jahren zugrunde.

Die Anwendung autonomer Roboter ist noch weitestgehend Zukunftsmusik (siehe oben I.). Zwar können aktuell eingesetzte Roboter bereits große Datenmengen aufnehmen und analysieren, in der Regel erfolgt diese Analyse aber zentralisiert und auch Verhaltensänderungen werden nur schrittweise in Form von Softwareupdates durchgeführt, bei denen eine zentrale Stelle aufgrund menschlichen Handelns die entsprechenden Änderungen überprüft und „,freigibt" (meist der Hersteller). ${ }^{18}$

Autonomie kann, wie auch Automatisierung, als graduelle Eigenschaft aufgefasst werden, d.h. ein System kann mehr oder weniger autonom agieren. ${ }^{19}$ So kann etwa ein Navigationssystem, das auf veränderte Umweltbedingungen in Form von Verkehrsmeldungen selbständig mit einer Verhaltensänderung (Änderung der Route) reagiert, bereits als in gewissem Grade autonom angesehen werden (der Algorithmus des Navigationssystems, nach dem dieses die Route berechnet, bleibt aber unverändert). Es scheint aber Einigkeit zu bestehen, dass hoch- oder vollautonome Systeme nicht nur einen quantitativen, sondern auch einen qualitativen Unterschied zu bestehenden automatischen Steuerungen darstellen. Einerseits ermöglichen sie, da sie definitionsgemäß nicht auf das Wissen ihrer Konstrukteure angewiesen sind, die Bewältigung unvorhergesehener komplexer Steuerungsaufgaben und versprechen so einen gewaltigen technischen Vorteil.

S. 141: „In embodied robotics werden Roboter mit sensorischer, motorischer und neuronaler Ausstattung untersucht, die im Laufe von Experimenten Erfahrungen sammeln, Verhaltensmuster und kognitive Fähigkeiten ausbilden."

17 Pratt (Fn. 8), S. 51 (52).

18 So jedenfalls die allgemeine Einschätzung im Rahmen der Tagung, die diesem Band zugrundeliegt.

19 Vgl. etwa Russell/Norvig (Fn. 4), S. 40: „To the extent that an agent relies on the prior knowledge of its designer rather than on its own percepts, we say that the agent lacks autonomy." (Hervorhebung durch den Verf.). Beer/Fisk/Rogers (Fn. 14), S. 74 (81 ff.), sprechen von ,levels of robot autonomy“. 
Andererseits ist dies zwingend mit dem Nachteil der fehlenden oder eingeschränkten Vorhersehbarkeit des Verhaltens verbunden.

\section{Automatisierungsrisiko}

Aus den dargestellten Besonderheiten der Robotertechnologie ergeben sich auch besondere Risiken, deren Bewältigung eine rechtliche Aufgabe ist, insbesondere des Sicherheitsrechts und des Haftungsrechts. Von besonderem rechtlichen Interesse sind dabei neuartige Risiken, also Risiken, die aufgrund der technischen Entwicklung entstanden sind (oder auch erst entstehen werden) und deren Auftreten dem Gesetzgeber bislang noch keine Zeit zum Handeln gelassen hat.

Sieht man von Missbrauchsszenarien, d.h. der gewollten Schädigung durch den Einsatz von Robotern, ab und betrachtet ungewollte Schäden, so lassen sich folgende besonderen „Roboterrisiken“ unterscheiden:

\section{Komplexitätsrisiko (Risiko computergesteuerter Maschinen)}

Das Komplexitätsrisiko ergibt sich aus dem Einsatz komplexer Maschinen und kann sich durch eine Fehlfunktion komplexer Algorithmen (Softwarefehler), unter Umständen auch durch eine Fehlfunktion komplexer Hardware, ergeben. ${ }^{20}$ Wie bereits angedeutet ist dieses Risiko nicht neuartig, sondern durch den Einsatz von Softwaresteuerungen oder den Betrieb komplexer Anlagen bekannt.

Während bestimmte sicherheitskritische Steuerungsaufgaben, wie etwa die Steuerung eines Kernkraftwerks, lange Zeit „by wire“ erfolgten, um Softwarerisiken hier auszuschließen, scheinen diese Bedenken gegenüber Softwaresteuerungen durch Fortschritte in der IT geringer zu werden. Zwar nimmt die Komplexität zumindest der Steuerung stets zu, allerdings scheint dies nicht mit einer Zunahme des Risikos einer Fehlfunktion verbunden zu sein. Unabhängig von der Beurteilung, die eine politische Aufgabe darstellt, handelt es sich aber jedenfalls um ein Risiko, das schon

20 M. R. Calo, Open Robotics, Maryland Law Review 70 (2011), S. 571 (593 ff.): „robots as physical PCs“. Zur Komplexität von Computern als Risikoquelle Baase, (Fn. 12), S. $405 \mathrm{ff}$. 
verhältnismäßig lange bekannt ist und jedenfalls nicht mehr als neuartiges Technikrisiko eingestuft werden kann.

Im Zusammenhang mit komplexen Steuerungen ist auch die Unterscheidung zwischen unternehmerischem und privatem Einsatz zu erwähnen. Systeme, deren Handhabung Fachwissen erfordert, können sich zumindest im unternehmerischen Einsatz als sicher genug erweisen. Kennzeichen hoch- und vollautomatisierter Steuerungen ist es aber gerade, dass diese ein menschliches Eingreifen gerade entbehrlich machen sollen. Damit kommt ihr Einsatz auch durch Verbraucher in Betracht. Allenfalls im Hinblick auf die Möglichkeit, Roboter von der Öffentlichkeit fernzuhalten (Schaffen von Barrieren, „Einsperren“ beweglicher Roboter), kann sich ein Unterschied zwischen unternehmerischem und privatem Handeln ergeben.

\section{Mobilitätsrisiko (Risiko durch Teilnahme am allgemeinen Verkehr)}

Eine weitere wichtige faktische Unterscheidung, die bei der Bestimmung der Verkehrspflichten im Umgang mit Robotern eine große Rolle spielen wird, ist diejenige nach dem physischen Umfeld des Robotereinsatzes. Dabei geht es nicht um die informationstechnische Vernetzung, sondern um die Frage, welchen körperlichen Barrieren sich die Roboter bei ihrem Einsatz gegenüber sehen. Geht es um Gefahren für Leib oder Leben, spielt die damit zusammenhängende Intensität des Umgangs mit Menschen eine Rolle. $^{21}$

21 Vgl. die Gefahrenabschätzung mit einer Einteilung nach Einsatzbereichen (Unterscheidung von Industrie- und Dienstleistungsrobotern) bzw. Intensität des Kontakts mit Menschen (z.B. Unterscheidung von Reinigungsroboter mit geringem Risikopotential, Dienstleistungsrobotern mit mittlerem Risikopotential und universell einsetzbaren humanoiden Robotern mit hohem Risikopotential) in einem Report des Japanese Robot Policy Committee, zitiert nach W. Yueh-Hsuan/H. ChienHsun/S. Chuen-Tsai, Toward the human-robot co-existence society: on safety intelligence for next generation robots, Int J Soc Robot 2009, S. 267 (268); Mainzer (Fn. 7), S. 145. 
a) Einsatz in geschlossener Umgebung oder Teilnahme am allgemeinen Verkehr

Werden Roboter innerhalb der eigenen Sphäre, also in einem geschlossenen räumlichen Bereich, eingesetzt, so fällt die Beherrschung der mit ihnen verbundenen Gefahren relativ leicht (allerdings verbleiben die bekannten Probleme des Arbeitnehmerschutzes beim Einsatz von Robotern).

Werden Roboter dagegen außerhalb der eigenen Sphäre eingesetzt und können sich frei in der Umwelt bewegen, so bedeutet dies ein wesentlich größeres Risiko für Dritte. Vergleichsweise konventionell ist die Interaktion von Robotern mit der Umwelt durch Datenaustausch. Insbesondere beim Einsatz von Computern zur Abgabe von Willenserklärungen ist dieser Aspekt der Rechtsordnung hinlänglich bekannt. ${ }^{22}$

Interessant ist, dass es einen technischen Präzedenzfall für die genannte Unterscheidung gibt, nämlich die Gentechnik. Das GenTG unterscheidet sicherheitsrechtlich klar zwischen gentechnischen Arbeiten in gentechnischen Anlagen, der Freisetzung zu Versuchszwecken und dem Inverkehrbringen. Ähnlich wie gentechnisch veränderte Organismen handelt es sich auch bei Robotern um Produkte, die eine gewisse Mobilität aufweisen. Allerdings können sich gentechnisch veränderte Organismen auch vermehren, was bei Robotern - noch - nicht der Fall ist.

\section{b) Kommerzieller oder privater Einsatz}

Nicht notwendig parallel zu der Unterscheidung von barrieregesichertem und freiem Einsatz verknüpft, verläuft die Unterscheidung von professionellem Einsatz und Einsatz durch Verbraucher. Industrieroboter sind zwar in der Regel auf einem Werksgelände gesichert, können aber z.B. auch die Auslieferung von Waren außerhalb der Unternehmenssphäre übernehmen (vgl. die Pläne zum Einsatz entsprechender Drohnen) ${ }^{23}$. Selbstfahrende Fahrzeuge, die von Verbrauchern eingesetzt werden, nehmen zwar am allgemeinen Verkehr teil, Verbraucher können aber auch Reinigungsroboter

22 Dazu G. Spindler, Zivilrechtliche Fragen bei Einsatz von Robotern, in: E. Hilgendorf (Hrsg.), Robotik im Kontext von Recht und Moral, Baden-Baden 2014; S. 63 (64f.).

23 Dazu http://www.heise.de/newsticker/meldung/Lieferroboter-und-Paketdrohnen-D ie-schoene-neue-Zustellwelt-3287196.html, zuletzt aufgerufen am 21.08.2016. 
einsetzen, die in Räumen „eingesperrt“ sind. Ein Grenzfall sind automatische Rasenmäher, die mehr oder weniger gut durch Barrieren gesichert sein können.

\section{Vernetzungsrisiko}

Ein weiteres neuartiges Risiko ergibt sich aus der angesprochenen Vernetzung moderner Roboter. Indem zahlreiche Mess-, aber auch Steuerungsdaten der Umgebung (d.h. andere Roboter desselben Herstellers, anderer privater Anbieter oder staatlicher Stellen) das Verhalten des Roboters beeinflussen, stellt sich die Frage der Zurechnung. ${ }^{24}$ Führt ein Steuerbefehl von außen zu einer Schädigung, so lässt sich die Kausalität möglicherweise zwar rechtlich, aber nicht mehr faktisch klar beschreiben.

\section{Autonomierisiko}

Ein besonderes und neuartiges Risiko ergibt sich aus dem prinzipiell unvorhersehbaren Verhalten selbstlernender Algorithmen. Sie sind Anlass für die aktuelle Debatte zum regulatorischen, haftungsrechtlichen und strafrechtlichen Umgang mit Robotern bzw. künstlicher Intelligenz. Die Autonomie als besondere Eigenschaft liegt Vergleichen mit Tieren, Kindern oder Angestellten und Vorschlägen für entsprechende rechtliche Analogieschlüsse zugrunde. ${ }^{25}$ Unklar ist, ob und wann autonome Systeme Selbstbewusstsein entwickeln werden und ihnen möglicherweise eine eigene Rechtspersönlichkeit zugebilligt werden muss. ${ }^{26}$ Aus regulierungs-

$24 \mathrm{Zu}$ den resultierenden haftungsrechtlichen Problemen M. Gruber, Zumutung und Zumutbarkeit von Verantwortung in Mensch-Maschine-Assoziationen. Ein Beitrag zur zivilrechtlichen Entwicklung der Roboterhaftung, in: E. Hilgendorf/J.-P. Günther (Hrsg.), Robotik und Gesetzgebung, Baden-Baden 2013, S. 145. Probleme der Zurechnung, die durch Robotertechnologie in besonderem Maße entstehen, sollen jedoch im vorliegenden Beitrag nicht ausführlich behandelt werden, dazu Gruber ,Zumutung und Zumutbarkeit (Fn. 24), S. 129; S. Horner/M. Kaulartz, Haftung 4.0 - Verschiebung des Sorgfaltsmaßstabs bei Herstellung und Nutzung autonomer Systeme, CR 2016, S. 7 ff.

25 Siehe unten D.IV.

26 Vgl. G. Teubner, Elektronische Agenten und grosse Menschenaffen: Zur Ausweitung des Akteursstatus in Recht und Politik, in: P. Becchi/Ch. B. Graber/M. Lumi- 
und haftungsrechtlicher Sicht stellen aber bereits autonome Systeme eine besondere Herausforderung dar.

Bei mobilen Robotern gewinnt die Autonomie besondere Brisanz. Einerseits wird gerade durch autonome Verhaltensweisen die Teilnahme am allgemeinen Verkehr erleichtert oder auch erst ermöglicht. Andererseits steigt dadurch aber auch das Risiko für die Öffentlichkeit. Der Einsatz voll-autonomer beweglicher Maschinen im öffentlichen Raum dürfte am oberen Ende der Risikoskala stehen.

\section{B. Haftungsrechtliche Grundlagen}

Roboter stellen technische Produkte dar. Führt ihr Einsatz zu Schäden, stellen sich die aus der Produkthaftung bekannten Probleme. ${ }^{27}$ Zunächst ist zu klären, wer als möglicher Haftender in Betracht kommt. Dabei ist insbesondere zwischen dem Inverkehrbringen und dem Betrieb von Robotern zu unterscheiden. Für die Haftung kommt es auf die Pflichtwidrigkeit an. Zum einen können Anwender Verkehrspflichten verletzen, zum andern können auch die Hersteller Verkehrspflichten verletzen, indem sie fehlerhafte Roboter in Verkehr bringen oder die Produktbeobachtungspflicht verletzen. Fehlt es an der Pflichtwidrigkeit, kommt nur eine Gefährdungshaftung in Betracht (sofern sie spezialgesetzlich angeordnet ist).

\section{Roboter als technische Produkte}

Roboter sind technische Produkte und damit verselbständigte Gefahrenquellen, durch die sich die spezifischen Risiken der Robotertechnologie verwirklichen können. Kommt es zu einem Schaden, haben Entwickler, Hersteller und Anwender in unterschiedlicher Mittelbarkeit bzw. Unmit-

nati (Hrsg.), Interdisziplinäre Wege der Grundlagenforschung, Zürich 2007, S. 1 ff.; M. Gruber, Was spricht gegen Maschinenrechte?, in: M. Gruber/J. Bung/S. Ziemann (Hrsg.), Autonome Automaten - Künstliche Körper und artifizielle Agenten in der technisierten Gesellschaft, 2. Aufl., Berlin 2015, S. 191 ff. Aus der Perspektive des Strafrechts S. Gless/Th. Weigend, Intelligente Agenten und das Strafrecht, ZStW 2014, S. 561 (566 ff.).

27 Ausführlich zur deliktischen Haftung beim Einsatz von Robotern Spindler (Fn. 22), S. 63 (68 ff.). 
telbarkeit dazu beigetragen. Dagegen sind Roboter selbst nicht als Rechtssubjekte anerkannt.

\section{Mögliche Verantwortliche im Umgang mit Robotertechnik}

Wie bei jeder Technologie kommen auch bei der Robotertechnik verschiedene Handelnde in Betracht, die im Umgang mit der Technologie abstrakte oder konkrete Risiken schaffen. Kommt es zum Schadenseintritt haben sie den Schaden mittelbar oder unmittelbar verursacht. Mögliche Ersatzpflichtige sind - in der Abfolge des jeweiligen Innovationszyklusses Entwickler, Hersteller, Händler oder Anwender. ${ }^{28}$ Hinzu kommt eine mögliche Haftung Dritter und de lege ferenda die Frage, ob auch Roboter selbst haften können.

\section{Produzentenseite (Inverkehrbringen von Robotern)}

Entwickler, Hersteller und Händler sind der Seite zuzuordnen, die für die Entstehung der Gefahrenquelle Roboter sorgen. Allen gemeinsam ist, dass sie Schäden, die durch den Einsatz von Robotern verursacht werden, nicht unmittelbar verursachen. In jedem Fall tritt noch das Handeln des Betreibers dazwischen.

\section{a) Entwickler}

Die Entwicklung und Verbreitung von Technologien (d.h. die Verbreitung des anwendbaren Wissens als solchem) lösen in der Regel keine haftungsrechtliche Verantwortlichkeit aus. ${ }^{29}$ Zwar gibt es durchaus Technologien, deren Geheimhaltung aus Sicherheitsgründen diskutiert wird, ${ }^{30}$ so dass bereits die Weitergabe anwendbaren Wissens als schadensverursachender

28 Vgl. E. Hilgendorf, Recht und autonome Maschinen - ein Problemaufriß, in: E. Hilgendorf/S. Hötitzsch (Hrsg.), Das Recht vor den Herausforderungen der modernen Technik, Baden-Baden 2015, S. 55 f.

29 Vgl. H. Zech, Gefährdungshaftung und neue Technologien, JZ 2013, S. 21 (28).

30 Z.B. genetische Veränderung von Krankheitserregern, vgl. D. Thurnherr, Biosecurity und Publikationsfreiheit, Bern 2014, S. 16 ff. (84 f.). 
Pflichtverstoß gewertet werden könnte, bei der zivilen Robotertechnologie dürfte dies aber nicht der Fall sein.

b) Hersteller

Der mittelbare Verursachungsbeitrag des Herstellers liegt der Produzenten- und Produkthaftung zugrunde. Der reine Entwickler einer bestimmten Technologie ist zwar in der Regel nicht rechtlich verantwortlich für die mit ihr verbundenen Risiken. Als Produzent trifft ihn jedoch die Produktund Produzentenhaftung. ${ }^{31}$

\section{c) Händler}

Ansprüche gegen den Händler ergeben sich zumeist nur, soweit vertragliche Beziehungen bestehen, d.h. beim Käufer. ${ }^{32}$ Hier bestehen bei Robotern keine Besonderheiten gegenüber anderen Produkten.

\section{Anwenderseite (Betrieb von Robotern)}

Von entscheidender Bedeutung für die Akzeptanz einer neuartigen Technologie wie der Robotics (und der aus ihr hervorgehenden neuen Produkte wie Pflegeroboter, Haushaltsroboter, selbststeuernde Fahrzeuge etc.) ist die Haftung bzw. Haftungsfreiheit der Anwender. Nur wenn sichergestellt ist, dass den Anwendern (zumindest, soweit es sich um Verbraucher handelt) keine übermäßigen Haftungsrisiken drohen, wird sich die Technik durchsetzen. Daher wird im Folgenden vor allem die Haftung der Produzenten und der Anwender untersucht.

31 Zur Ausnahme bei Entwicklungsrisiken weiter unten (III.1.a, IV.).

32 Zur Vertragshaftung bei Produkten H. Kötz/G. Wagner, Deliktsrecht, 12. Aufl., München 2013, Rn. 606 f., m.w.N. 
a) Kommerzieller Anwender

Ein großer Unterschied ergibt sich, je nachdem, ob Roboter im unternehmerischen Bereich oder im privaten Bereich eingesetzt werden. Der professionelle Einsatz erlaubt sowohl eine bessere faktische Beherrschung der mit ihm verbundenen Risiken als auch eine bessere ökonomische Absicherung der damit verbundenen Haftungsrisiken. Letzteres ergibt sich bereits aus dem Umstand, dass ein Unternehmer Risiken einkalkulieren und auf seine Kunden umlegen kann. Gesetzlich könnte auch eine besondere Sicherstellung durch eine Verpflichtung zum Vorhalten eines bestimmten Haftungsfonds oder eine Versicherungspflicht bewirkt werden. ${ }^{33}$

b) Verbraucher

Geht es um den Einsatz von Robotern durch Verbraucher, so muss der Einsatz auch mit der lediglichen verbraucherüblichen Sorgfalt möglich sein und eine Abdeckung der Risiken durch persönliche Haftung des Verbrauchers ohne Versicherungsdeckung scheidet aus (Akzeptanzargument). Auf das gesetzliche Modell des Betriebs von Kraftfahrzeugen (Typenzulassung, Zulassungspflicht, Gefährdungshaftung und Pflichtversicherung) wird noch zurückzukommen sein.

\section{c) Roboter als Haftungssubjekt}

Ein weiteres besonders Thema, das an die Grundfesten der Rechtsordnung rührt, ist die Frage, ob auch die Roboter selbst haften können bzw. sollen. Dies setzt notwendig voraus, dass eine eigene Rechtspersönlichkeit von Robotern anerkannt wird (und ein Haftungssubstrat besteht). Am Ende des Beitrags soll auf diese Frage kurz eingegangen werden. Es gilt vor allem zwischen Robotern als neuartigen natürlichen Personen und aus dem Gesellschaftsrecht hinlänglich bekannten juristischen Personen zu unterscheiden. Die zentrale Frage, ob Roboter Persönlichkeit besitzen können, ist eine Frage der Rechtsphilosophie. 


\section{Haftung Dritter}

Als gesondertes Thema ist die mögliche Haftung Dritter zu sehen, insbesondere bei Eingriffen in die Funktionsfähigkeit von Robotern. Dies betrifft in erster Linie Missbrauchsszenarien (Frage der security/Angriffssicherheit im Gegensatz zur safety/Betriebssicherheit, die sich auf Risiken des regulären Betriebs bezieht ${ }^{34}$ ), wie sie im Zusammenhang mit jeder neuartigen Technologie diskutiert werden können. Der Missbrauch von Robotern wird wie geschildert, vor allem durch die Vernetzung erleichtert.

\section{Haftung für pflichtwidriges Verhalten (Pflichten im Umgang mit Robotertechnologie)}

Sowohl den Hersteller als auch den Anwender können im Umgang mit Robotern bestimmte Verhaltenspflichten treffen. Diese folgen aus dem $\mathrm{Zu}-$ sammenspiel von Verkehrspflichten und absolut geschützen Rechtsgütern oder aus einem Schutzgesetz. Im Kern geht es immer um die Frage, ob der Handelnde das erlaubte Risiko überschritten hat. ${ }^{35}$ Unterschiede ergeben sich bei der Kompetenz zur Bestimmung dieser Grenze.

Liegt pflichtwidriges Handeln vor, kommt nicht nur ein Schadensersatz nach $\S 823$ BGB in Betracht, sondern auch negatorischer Rechtsschutz nach $§ 1004$ I BGB. Bei einer konkreten Gefährdung von Rechtsgütern Dritter oder von Schutzgesetzen können Dritten Unterlassungsansprüche zustehen.

\section{Haftung wegen Verletzung geschützter Rechtsgüter (\$ 823 Abs. 1 BGB)}

Die Beeinträchtigung fremder geschützter Rechtsgüter führt zur Rechtswidrigkeit, wenn sie unter Verstoß gegen Verkehrspflichten erfolgt. ${ }^{36}$ Bei einer unmittelbaren Beeinträchtigung wird dies ohne weiteres angenom-

34 Vgl. Calo, (Fn. 20), S. 571 (594).

35 Kötz/Wagner (Fn. 32), Rn. 111 (169 f.).

36 Zur Haftung nach §823 Abs. 1 BGB bei Schäden durch Roboter Spindler, (Fn. 22), S. 63 (69 ff.); J. Hanisch, Zivilrechtliche Haftungskonzepte für Robotik, in: Hilgendorf (Hrsg.), Robotik im Kontext von Recht und Moral, Baden-Baden 2014, S. 27 (34). 
men, bei einer mittelbaren Beeinträchtigung wird gesondert geprüft, ob der Handelnde die objektiv erforderlichen Maßnahmen zur Verhinderung eines Schadens ergriffen hat. ${ }^{37}$ Dies ist mit der Prüfung der objektiven Sorgfalt im Rahmen des Verschuldens identisch. ${ }^{38}$

Als beeinträchtigte Rechtsgüter kommen Leib und Leben, Eigentum, aber auch informationsbezogene Persönlichkeitsrechte (Recht am eigenen Bild, Datenschutz, ${ }^{39}$ allgemeines Persönlichkeitsrecht) in Betracht. Sie genießen deliktischen Rechtsschutz nach $\S 823$ Abs. 1 BGB. Häufig werden dagegen auch reine Vermögensschäden auftreten, die zwar unter Umständen bei Vorsatz ersatzfähig ( $\$ 826$ BGB), ansonsten aber regelmäßig hinzunehmen sind.

Die Bestimmung der erforderlichen Sorgfalt erfolgt durch die Rechtsprechung. Verlangt wird die Vornahme möglicher und zumutbarer Sicherheitsmaßnahmen, wobei der Bestimmung des Zumutbaren eine KostenNutzen-Abwägung zugrunde liegt. ${ }^{40}$ Geht es um Gefahren für hochrangige Rechtsgüter wie Leib und Leben, die von den Betroffenen nicht oder nur mit hohem Aufwand abgewehrt werden können, sind die Anforderungen entsprechend hoch. Sind bestimmte Verhaltensweisen erwünscht, können möglicherweise auftretende Schäden aber auch hinzunehmen sein (allgemeines Lebensrisiko).

\section{a) Hersteller}

Den Hersteller treffen die für die allgemeine Produkthaftung entwickelten Pflichten. ${ }^{41}$ Voraussetzung für die Annahme eines Pflichtverstoßes ist immer das Vorliegen eines Produktfehlers (dazu sogleich), d.h. eine nicht der erforderlichen Sorgfalt entsprechende Konstruktion, Fabrikation, Instruk-

37 Kritisch zu der traditionellen Unterscheidung zwischen unmittelbarer und mittelbarer Beeinträchtigung Kötz/Wagner (Fn. 32), Rn. 109. Es liegt nahe, davon auszugehen, dass auch bei einer kausalen Beeinträchtigung ohne Zwischenursachen die Verletzung von Verkehrspflichten zu fordern ist, wegen der Unmittelbarkeit der Beeinträchtigung aber auch die Anforderungen entsprechend hoch sind.

38 Kötz/Wagner (Fn. 32), Rn. 128.

39 Zum Datenschutz bzw. einem Recht an den eigenen persönlichen Daten als sonstiges Recht im Sinne des $\S 823$ Abs. 1 BGB; H. Zech, Information als Schutzgegenstand, Tübingen 2012, S. $217 \mathrm{ff}$.

40 MüKo-Wagner, § 823 BGB, Rn. 316 (337 ff.).

41 Spindler (Fn. 22), S. 63 (72). 
tion. Die Fehlerhaftigkeit führt dann zur Vermutung eines objektiv pflichtwidrigen Handelns. Die wesentliche Funktion des Abstellens auf Produktfehler besteht darin, dass ein Geschädigter zum Beweis eines pflichtwidrigen Handelns des Herstellers nicht hinter die Werkstore blicken muss.

Insbesondere Entwicklungsrisiken (also Risiken, die nach dem Stand von Wissenschaft und Technik zum Zeitpunkt des Inverkehrbringens nicht erkennbar waren) erlauben jedoch eine Entlastung. ${ }^{42}$ Die Verhinderung des Unerkennbaren ist unzumutbar. Im Bereich der Robotertechnologie wird man jedoch die unter A. dargestellten Risiken als bekannt bezeichnen müssen. Dies gilt auch für Autonomierisiken, die dem Grunde nach vorhersehbar sind (dazu unter D.I).

Eine besondere Herstellerpflicht ist darüberhinaus die Produktbeobachtung. Produkte stellen eine verselbständigte Risikoquelle dar. Mit dem Inverkehrbringen setzt der Produzent ein Risiko, das er nicht mehr vollständig beherrschen kann, das aber als Produkt unter Umständen eine lange Zeit bestehen bleibt und durch aktives Handeln (Produktrückruf etc.) auch beeinflusst werden kann. Daraus leitet sich die besondere Bedeutung der Produktbeobachtung ab.

\section{b) Betreiber}

Bei den Betreiberpflichten geht es vor allem um die Verschuldensprüfung. Wurde die erforderliche Sorgfalt gewahrt, scheitert die Haftung nach $\S 823$ Abs. 1 BGB mangels Verschulden. Daneben gibt es Fallkonstellationen, in denen auch der Einsatz von Robotern nur mittelbar zu einer Schädigung führt (das Inverkehrbringen dann sozusagen „doppelt“ mittelbar), womit eine Verkehrspflichtverletzung nicht ohne weiteres angenommen werden kann.

Ein Zukunftsszenario liegt der Frage zugrunde, ob man Roboter als eigene Rechtspersonen auffassen kann. Dann wäre eine Schädigung durch den Einsatz von Robotern nur mittelbar verursacht. Allerdings wäre dann auch ohne weiteres $\S 831$ BGB anzuwenden.

42 Kötz/Wagner(Fn. 32), Rn. 624. 


\section{Verstoß gegen Verbotsgesetze ( $§ 823$ Abs. 2 BGB)}

Denkbar ist auch eine Haftung wegen Schutzgesetzverletzung nach $\S 823 \mathrm{Abs}$. 2 BGB. Die Bestimmung der Sorgfaltsanforderungen wird hier durch die Legislative vorgenommen. Allerdings bestehen bislang im Bereich der Robotertechnologie noch keine entsprechenden gesetzlichen Regelungen. ${ }^{43}$

Einen Sonderfall stellen selbstfahrende Fahrzeuge dar. Hier findet das Zulassungsrecht (FZV) und Verhaltensrecht (StVO) für Kraftfahrzeuge Anwendung, das noch entsprechend angepasst werden soll. ${ }^{44}$

\section{Einfluss technischer Normung und gesetzgeberische Gestaltungsmöglichkeiten}

Bei der Bestimmung der objektiv erforderlichen Sorgfalt, sei es im Rahmen der Verschuldensprüfung oder im Rahmen der Bestimmung von Verkehrspflichten, spielen technische Normen eine große Rolle, wenn sie auch das Gericht nicht binden. ${ }^{45}$ Für Roboter gibt es bereits Standards. ${ }^{46}$ Ihr Vorteil besteht in einer flexibleren Anpassung als durch Gesetzgebung.

Eine andere Frage ist die Bedeutung von öffentlich-rechtlichen Normen für die Beurteilung der zivilrechtlich erforderlichen Sorgfalt. Unproblematisch können sie Schutzgestze im Sinne des $\S 823$ Abs. 2 BGB enthalten. Darüber hinaus sollten sie aber auch für die Beurteilung von Verkehrsbzw. Sorgfaltspflichten bindend sein. ${ }^{47}$ Dies ergibt sich weniger aus dem Argument der Einheit der Rechtsordnung. Gerade bei der Einführung neuer Technologien ist es aber eine politische Entscheidung, ob und unter welchen Bedingungen bestimmte Risiken eingegangen werden sollen. Sicherheitsrechtliche Regelungen sind das passende Vehikel zur rechtlichen Umsetzung solcher Entscheidungen. Dann müssen sie aber auch in der Lage sein, erwünschtes Verhalten zu legitimieren - nicht nur für das öf-

43 Spindler (Fn. 22), S. 63 (77 f).; vgl. Hanisch (Fn. 36), S. 27 (35).

44 Gasser (Fn. 14), S. 51 ff.

45 MüKo-Wagner, § 823 BGB, Rn. 360 ff.

46 EN ISO 10218-1 und -2: Industrieroboter - Sicherheitsanforderungen (Teil 1 Roboter, Teil 2 Robotersysteme und Integration); vgl. Spindler (Fn. 22), S. 63 (71); W. Kohte, Arbeitsschutz in der digitalen Arbeitswelt, NZA 2015, S. 1417 (1419f.).

47 Anderer Ansicht MüKo-Wagner, § 823 BGB, Rn. 359 f. 
fentliche Recht, sondern auch für das Privatrecht. Nur durch die resultierende Rechtssicherheit wird die Einführung neuer Technologien ermöglicht, die erwünscht (da nützlich), aber auch riskant sind (zur flankierenden Einführung von Gefährdungshaftungsregelungen unten E).

\section{Produkthaftung (Haftung nach ProdHaftG)}

Auch die Produkthaftung ist als Haftung für pflichtwidriges Handeln zu qualifizieren. Ein Produkt hat einen Fehler, wenn es nicht die Sicherheit bietet, die unter Berücksichtigung aller Umstände berechtigterweise erwartet werden kann (vgl. § 3 Abs. 1 ProdHaftG). Auch hier hat sich die Unterscheidung von Konstruktions-, Fabrikations- und Instruktionsfehlern herausgebildet. Zwar kennt das ProdHaftG kein Verschuldenserfordernis, es setzt aber mit dem Produktfehler einen Pflichtverstoß voraus. ${ }^{48}$

Entwicklungsfehler führen nach $\S 1$ II Nr. 5 ProdHaftG zum Ausschluss der Ersatzpflicht des Herstellers nach ProdHaftG. ${ }^{49}$ Sofern der Hersteller über kein besseres Wissen verfügen kann als der Verbraucher, soll er nicht haften. Die Entlastung für Entwicklungsrisiken ist ebenfalls als Verschuldenselement zu werten. ${ }^{50}$ Wie bereits ausgeführt, dürfte jedoch die Ausbildung im Einzelnen nicht vorhersehbarer Verhaltensweisen durch autonome Roboter gerade nicht als Entwicklungsfehler anzusehen sein, weil sie im Grunde vorhersehbar ist. Astrid Auer-Reinsdorff spricht anschaulich von „Anlernfehlern“. 51

Auch dem Regelungszweck nach geht es dem ProdHaftG nicht um die Zuweisung der spezifischen Risiken neuer Technologien (oder anderer besonderer Risiken), sondern nur um die sorgfältige Herstellung von Produkten. Gerhard Wagner spricht von einem semantischen Trick. ${ }^{52}$ Steue-

48 Kötz/Wagner (Fn. 32), Rn. 614: „Verschuldensprüfung de facto in den Fehlerbegriff verlagert“. Das Inverkehrbringen fehlerhafter Produkte stellt überdies auch einen Verstoß gegen sicherheitsrechtliche Pflichten dar, vgl. § 3 ProdSG.

49 Art. 7 lit. e Richtlinie 85/374/EWG ermöglicht eine Einbeziehung von Entwicklungsrisiken, von der jedoch nur wenige EU-Mitgliedstaaten Gebrauch gemacht haben.

50 MüKo-Wagner, Einleitung ProdHaftG, Rn. 15.

51 A. Auer-Reinsdorff, persönlicher Vortrag am 28.10.2015, München.

52 MüKo-Wagner, Einleitung ProdHaftG, Rn. 17: „Zurechnungsgrund der Gefährdungshaftung ist die zum eigenen Nutzen ausgeübte Kontrolle einer Quelle erhöhter Gefahr, bei der sich Schäden erheblichen Umfangs auch bei Anwendung der im 
rungsinstrument der Wahl für Technikrisiken sind echte Gefährdungshaftungstatbestände (nicht aber das ProdHaftG, das ein pflichtwidriges Inverkehrbringen voraussetzt). Auf die Funktion solcher Haftungsregelungen wird unter E.I noch einmal genauer eingegangen.

\section{Gefährdungshaftung}

Eine Gefährdungshaftung greift nur dort ein, wo ein entsprechender Spezialtatbestand vorhanden ist. Für Roboter gibt es eine solche spezielle Gefährdungshaftung bislang noch nicht. Auch Analogien zu bestehenden Tatbeständen werden zumindest von der Rechtsprechung abgelehnt, sollen jedoch unter D.IV genauer beleuchtet werden. ${ }^{53}$

Unter Umständen greifen aber andere Gefährdungshaftungen ein, insbesondere $\S 7 \mathrm{StVG}$ für selbststeuernde Fahrzeuge. ${ }^{54}$ Denkbar wäre auch eine Haftung nach $\S 33$ LuftVG beim Einsatz von Drohnen, nach $\S 1$ HaftPflG bei automatisierten Schienenbahnen oder nach $\S 114$ ff. BBergG bei automatisierten Einrichtungen im Bergbau). Alle diese Tatbestände zielen jedoch nicht auf die spezifischen Risiken der Robotertechnologie, also Automatisierungs- und Autonomierisiken) ab.

Verkehr erforderlichen Sorgfalt nicht vermeiden lassen [...]. Als solche Gefahrenquelle mit selbst bei Beachtung aller möglichen und wirtschaftlich zumutbaren Sicherheitsmaßnahmen besonderer Schadensneigung stellt sich die Warenproduktion gerade nicht dar [...]. [Der Hersteller] haftet nicht deshalb, weil ihm das nach Ergreifung aller Sorgfaltsmaßnahmen verbleibende Schadensrisiko auferlegt werden soll, sondern deshalb, weil er es versäumt hat, mögliche und zumutbare Sicherheitsmaßnahmen zu ergreifen. Dieser Befund lässt sich auch nicht mit dem semantischen Trick kaschieren, kurzerhand das Inverkehrbringen eines fehlerhaften Produkts als Gefahrenquelle auszugeben, denn dann wird der Fehler in den Begriff der Gefahrenquelle integriert, so dass Delikts- und Gefährdungshaftung eins werden: Knüpft die vermeintliche Gefährdungshaftung an einen Sorgfaltspflichtverstoß an, dann ist sie faktisch Verschuldenshaftung im Sinne einer Haftung für verkehrswidriges Verhalten."

53 Für eine Gesamtanalogie zu den bestehenden Gefährdungshaftungstatbeständen Kötz/Wagner (Fn. 32), Rn. 514.

54 C. Bewersdorf, Zulassung und Haftung bei Fahrerassistenzsystemen im Strassenverkehr, Berlin 2005, S. $101 \mathrm{ff}$; M. F. Lohmann, Liability Issues Concerning SelfDriving Vehicles, EJRR 2/2016, S. 335 (336f.); dies. Automatisierte Fahrzeuge (Fn. 14), S. 211 ff. (zum Schweizer Recht). 
Eine Gefährdungshaftung für Roboter ist also vor allem eine Frage de lege ferenda, wobei auf den Steuerungszweck von Technik-Gefährdungshaftungstatbeständen noch ausführlicher eingegangen werden muss (dazu unter E.).

\section{Haftung für hoch- und vollautomatisierte Roboter (Automatisierungsrisiko)}

Der Einsatz hoch- und vollautomatisierter Maschinen ist bereits Realität. Ihre weite Verbreitung auch im privaten Bereich steht in den nächsten Jahren bevor. Das gilt auch für mobile Roboter, wobei der erste Anwendungsfall von großer wirtschaftlicher Bedeutung selbststeuernde Fahrzeuge sein dürften.

\section{Tatbestandliche Kriterien für eine Beurteilung des Automatisierungsrisikos}

Das spezifische Risiko hoch- und vollautomatisierter Roboter liegt in der entsprechend automatisierten Steuerung von Aktuatoren. Handelt es sich um bewegliche Maschinen, die am öffentlichen Verkehr teilnehmen, wird dieses Risiko vergrößert.

Sowohl für die Beurteilung der Haftung (und insbesondere der Verhaltenspflichten) de lege lata als auch für Einführung möglicher gesetzlicher Regelungen ist es sinnvoll, wichtige Parameter für die Risikobeurteilung $\mathrm{zu}$ benennen. Aus rechtlicher Sicht kann man das Risiko der jeweiligen Maschine bzw. des jeweiligen Roboters (als einzelnes Produkt oder als Typ) nach verschiedenen Kriterien einteilen:

\section{Einteilung nach Automatisierungsgrad}

Zunächst kann man eine Einteilung nach dem Automatisierungsgrad und der korrespondierenden Eingriffsmöglichkeit des Bedieners vornehmen. Diese Einteilung spielt z.B. für automatisierte Fahrzeuge eine große Rolle.

Geräte mit einem geringen Automatisierungsgrad (Teilautomatisierung) wird man bereits nicht als Roboter ansprechen müssen. Hier greifen die Überlegungen $\mathrm{zu}$ den rechtlichen Folgen des besonderen Automatisie- 
rungsrisikos nicht. Ein Beispiel sind die bereits zugelassenen Fahrzeuge mit Assistenzsystemen.

\section{Größe und Leistung}

Ein weiteres Kriterium ist die Größe der Roboter. Kleinere Roboter stellen wegen ihrer geringeren Kraftentfaltung grundsätzlich ein kleineres Risiko dar (Ausnahmen sind jedoch denkbar). Eine solche Abgrenzung gibt es z.B. bei Drohnen, deren Nutzung bis zu einem Gesamtgewicht von $5 \mathrm{~kg}$ derzeit nicht erlaubnispflichtig ist ( $\$ 20$ Abs. 1 Nr. 1 lit. a LuftVO).

Auch die Energie, die ein Roboter freisetzen kann bzw. seine mögliche Leistung (Energieumsatz pro Zeit), spielt eine Rolle. Ein Extrembeispiel sind Waffensysteme, die auch bei geringer Größe hohe Energiemengen freisetzen können und deshalb besonders gefährlich sind.

\section{Mobilität}

Wichtig ist auch die Unterscheidung, ob es sich um ein immobiles Gerät oder zumindest um ein Gerät handelt, das nur in umgrenzten Räumen oder innerhalb von Barrieren betrieben wird, oder ob sich der Roboter frei im öffentlichen Raum bewegen soll.

\section{Sachkunde des Nutzers}

Schließlich kann man danach unterscheiden, ob Roboter an professionelle Nutzer abgegeben bzw. von diesen betreiben werden oder ob es sich um ein Verbrauchergerät handelt.

Auch im Verbraucherbereich kann eine besondere Sachkunde des Nutzers bzw. Bedieners sichergestellt werden, indem entsprechende sicherheitsrechtliche Regelungen geschaffen werden. Ein Beispiel im Bereich der nicht-automatisierten Technik ist die Führerscheinpflicht für das Fahren von Kraftfahrzeugen. Hier kann, wie auch bei Kraftfahrzeugen, zwischen Bediener (beim Fahrzeug Fahrer) und Halter unterschieden werden. 


\section{Inverkehrbringen hoch- und vollautomatisierter Maschinen}

Als Rechtsgrundlagen für eine Haftung der Hersteller kommen wie bereits erwähnt sowohl $\S 823$ Abs. 1 BGB in der Ausgestaltung durch die BGHRechtsprechung zur Produzentenhaftung als auch das Produkthaftpflichtgesetz in Betracht. ${ }^{55}$ Beide setzen die Pflichtwidrigkeit des Robotervertriebs voraus (zumindest in der Gestalt der Fehlerhaftigkeit). De lege ferenda könnte man wie schon erwähnt über die Einführung einer eigenen Gefährdungshaftung für den Vertrieb von Robotern nachdenken. ${ }^{56}$

\section{Inverkehrbringen automatisierter Maschinen, insbesondere selbststeuernder Fahrzeuge, entspricht grundsätzlich der üblichen Sorgfalt}

Ebenso wie das Inverkehrbringen konventioneller Fahrzeuge grundsätzlich keinen Verkehrspflichtverstoß darstellt, dürfte dies auch für automatisierte Fahrzeuge gelten. Kommt es durch die Zulassung einer automatisierten Steuerung (z.B. im Straßenverkehr) zu einer Verringerung von Unfallzahlen, so ist ein automatisiertes Gerät sogar günstiger zu beurteilen als ein nicht-automatisiertes.

Die Diskussion um „tödliche Algorithmen“"57 oder ethische Dilemmata bei „Entscheidungen“ automatischer Fahrzeuge ist aus Sicht des Zivilrechts von geringer Bedeutung, da es nicht um unmittelbar kausales schadensverursachendes Handeln geht (wie beim Anwender, den aber mangels Sorgfaltspflichtverstoßes keine Haftung trifft), sondern um mittelbar kausales Handeln (Inverkehrbringen eines Produkts), das nur bei Verkehrs-

55 Bewersdorf (Fn. 54), S. 133 ff.; Gruber, Zumutung und Zumutbarkeit (Fn. 24), S. 143 ff.; ders. Bioinformationsrecht - Zur Persönlichkeitsentfaltung des Menschen in technisierter Verfassung, Tübingen 2015, S. 276 ff.; Spindler (Fn. 22), S. 63 (69 ff.); Th. Klindt, Fahrzeugautomatisierung unter dem Blickwinkel des Produktsicherheits- und Produkthaftungsrechts, in: Hilgendorf/Hötitzsch/Lutz (Hrsg.), Rechtliche Aspekte automatisierter Fahrzeuge, Baden-Baden 2015, S. 62 f.; Günther, Roboter und rechtliche Verantwortung: (Fn. 16), S. 144 ff.. Zum Schweizer Recht Lohmann, Automatisierte Fahrzeuge (Fn. 14), S. 297 ff.

56 Dazu unter E.III.

57 Vgl. S. Schuppli, Deadly algorithms - Can legal codes hold software accountable for code that kills?, Radical Philosophy 187 (2014), S. 1, (verwendet den Begriff „deadly algorithms“). 
pflichtwidrigkeit zur Haftung führt. Daher ist es auch im Hinblick auf die berührten wichtigen Rechtsgüter wie Leib und Leben vertretbar, solche Technologien einzusetzen, wenn es zu einer Reduktion des ohnehin bereits erlaubten Risikos kommt. Der Einsatz solcher Algorithmen bedeutet dann eine schlichte Risikoverringerung gegenüber dem Vertrieb herkömmlicher Maschinen (Fahrzeuge), welcher ein von der Rechtsordnung wegen seiner Nützlichkeit geduldetes Risiko darstellt.

\section{Mögliche gesetzgeberische Klarstellung}

Wegen der Wichtigkeit von Algorithmen zur Steuerung von Fahrzeugen, die auch zwischen menschlichen Leben abwägen, kann man die Zulässigkeit ihres Einsatzes und die entsprechenden Anforderungen gesetzlich regeln (möglicherweise muss man das auch aufgrund verfassungsrechtlicher Vorgaben). Hält sich der Roboterhersteller an die entsprechenden Vorgaben, so bleibt er haftungsfrei. Einen entsprechenden Vorstoß plant offenbar die Bundesregierung. ${ }^{58}$

\section{Produktbeobachtung}

Wie bei allen technischen Produkten spielt die Produktbeobachtungspflicht eine große Rolle. Bei Robotern führt sie nicht nur zu den üblichen Warn- und Rückrufpflichten, sondern vor allem auch zu einer Pflicht, nötige und zumutbare Softwareupdates zur Verfügung zu stellen.

\section{Kommerzieller Einsatz}

\section{Haftung nach § 823 Abs. $1 B G B$}

Wegen der entsprechenden Möglichkeiten zur Schadensverhütung bestehen beim unternehmerischen Einsatz von Robotern höhere Sorgfaltspflichten als bei der privaten Verwendung. Da auch bei einem Einsatz immobiler oder in ihrer Bewegung räumlich eingegrenzter Roboter zumindest Arbeitnehmer (des Betreiberunternehmens oder eines anderen Unterneh-

58 FAZ vom 11.7.2016, S. 19, Ethikkommission für Roboterautos. 
mens, in dem die Roboter eingesetzt werden) dem Risiko ausgesetzt sind, handelt es sich vor allem um ein Thema der Arbeitssicherheit. ${ }^{59}$ Unfälle mit Industrierobotern der ersten Generation haben aber dazu geführt, dass es bereits genaue Verhaltensstandards gibt.

Bei einem Einsatz im öffentlichen Raum, wo auch nicht geschulte Dritte mit den Robotern in Kontakt kommen, sind besonders hohe Anforderungen an die Verkehrspflichten zu stellen. Dabei darf freilich eine nützliche Entwicklung nicht verhindert werden, was bei der Abwägung zu berücksichtigen ist.

Im Ergebnis ist daher festzuhalten, dass der Einsatz von Robotern jedenfalls nicht ohne besondere Umstände (unsorgfältige Schulung von Mitarbeitern, Wartung, Updates etc.) zu einer Verschuldenshaftung führt. Grundsätzlich gilt aber: Werden die Anforderungen erfüllt, so trifft den Betreiber nach geltendem Recht keine Haftung.

\section{Analoge Anwendung des $\S 831$ Abs. 1 BGB (Roboter als Angestellte)}

Wegen der grundsätzlichen Pflichtkonformität des Einsatzes automatisierter Maschinen führt auch eine mögliche Haftung in Analogie zur Geschäftsherrenhaftung nach $\S 831$ Abs. 1 BGB $^{60}$ zu keinem anderen Ergebnis. Zwar wäre es durchaus denkbar, auch ohne Rechtspersönlichkeit von Robotern eine Analogie zu Verrichtungsgehilfen zu bilden. Im Kern handelt es sich um eine Haftung für vermutetes eigenes Verschulden beim Einsatz des Verrichtungsgehilfen. Daher ist auch die eigene Verschuldensfähigkeit des Verrichtungsgehilfen nicht erforderlich. ${ }^{61}$ Dem Einsetzenden wird aber aus den unter 1. genannten Gründen der Entlastungsbeweis in der Regel gelingen.

59 Dazu Kohte, (Fn. 46), S. 1417 (1419 f.).

60 Dazu R. John, Haftung für künstliche Intelligenz - Rechtliche Beurteilung des Einsatzes intelligenter Softwareagenten im E-Commerce, Hamburg 2007, S. 272 ff.; Günther, Roboter und rechtliche Verantwortung (Fn. 16), S. 134. Zum Schweizer Recht bereits K. Spiro, Die Haftung für Erfüllungsgehilfen, Bern 1984, S. 209 ff. (Einsatz von Maschinen).

61 MüKo-Wagner, § 831 BGB, Rn. 31. 


\section{Einsatz durch Verbraucher}

Soweit der Anwender eines Roboters nicht die pflichtgemäße Sorgfalt walten lässt, haftet er nach $\S 823$ Abs. 1 BGB. Allerdings dürften für nicht-unternehmerische Anwender die Anforderungen niedrig anzusetzen sein. ${ }^{62}$ Wie bereits erwähnt wird es auch zu einer breitflächigen Anwendung von Robotern durch Verbraucher nur dann kommen, wenn die erforderlichen Sorgfaltsstandards entsprechend klar und niedrig angesetzt sind.

Im Ergebnis kommt eine Haftung daher nur dann in Betracht, wenn besondere roboterspezifische Anwenderpflichten verletzt werden, wie etwa eine Pflicht zur Vornahme der vom Hersteller angebotenen Softwareupdates oder ähnliches. Eine entsprechende gesetzgeberische Klarstellung ist geplant. ${ }^{63}$

\section{Haftung für autonome Roboter (Autonomierisiko)}

Anders als der Einsatz hoch- und vollautomatisierter Roboter ohne Autonomie ist der Einsatz autonomer Roboter nach geltendem Recht grundsätzlich als pflichtwidrig zu beurteilen. Grund ist die mangelnde Beherrschbarkeit und Abschätzbarkeit des Autonomierisikos, jedenfalls nach aktuellem Kenntnisstand. Durch gesetzliche Regelung könnte der Einsatz zulässig gemacht werden. Dann stellt sich aber wegen des besonderen Risikos die Frage nach einer möglichen Gefährdungshaftung der Anwender. ${ }^{64}$

\section{Risikobeurteilung bei autonomen Maschinen}

Autonome Roboter zeichnen sich durch das unter A.III dargestellte besondere Autonomierisiko aus. Die Unvorhersehbarkeit des Verhaltens autonomer Maschinen macht auch die Risikobeurteilung schwerer. Der Umgang

62 Vgl. Spindler (Fn. 22), S. 63 (65 f.); Horner/Kaulartz (Fn. 24), S. 7 (8).

63 FAZ vom 11.7.2016, S. 19, Ethikkommission für Roboterautos: „,[Bundesverkehrsminister] Dobrindt zufolge soll sichergestellt werden, dass Autofahrer bei ordnungsgemäßer Nutzung des Autopiloten ihre Sorgfaltspflicht nicht verletzen und ihnen so keine zusätzlichen Haftungsrisiken entstehen können.“

64 Dazu unter E.IV. 
mit autonomen Maschinen kann sich auch nicht auf eine lange Erfahrung stützen, wie das z.B. bei Tieren der Fall ist. Zwar mag eine bessere Beurteilung durch Versuche möglich werden, der derzeitige Kenntnisstand ist aber sicherlich noch nicht ausreichend.

Das Autonomierisiko ist zwar schwer zu beurteilen, d.h. für den konkreten Einsatz einer konkreten Maschine als Produkt von Schadenshöhe und Eintrittswahrscheinlichkeit abzuschätzen. Es ist aber unschwer als solches zu erkennen. Ob eine Maschine autonom agieren kann, ist sehr leicht festzustellen. Allenfalls bedarf es einer Abgrenzung, ab welchem Grad von Autonmie ein rechtlich relevantes Autonomierisiko vorliegt. Verändern Maschinen die Ihrem Verhalten zugrundeliegenden Algorithmen selbstständig, dürfte dies zu bejahen sein.

Weitere beeinflussende Faktoren können die gleichen sein, die bereits bei automatisierten Maschinen angesprochen wurden (C.I), insbesondere also die Größe bzw. mögliche Leistung der Maschine, ihre Mobilität und die Sachkunde des Einsetzenden (Halters).

\section{Inverkehrbringen autonomer Roboter}

\section{Inverkehrbringen autonomer Roboter als Sorgfaltspflichtverletzung bzw. Autonomie als Konstruktionsfehler de lege lata}

Vieles spricht dafür, autonome Roboter wegen der Unvorhersehbarkeit ihres Verhaltens als per se fehlerhaft einzustufen (Konstruktionsfehler). Das Inverkehrbringen autonomer Maschinen wäre damit automatisch ein pflichtwidriges Verhalten. Im Rahmen des $\S 823$ Abs. 1 BGB läge eine relevante mittelbare Rechtsgutsverletzung vor, da diese Pflichtwidrigkeit das Verletzen einer Verkehrspflicht bedeutet.

Es liegt auch kein Entwicklungsrisiko bzw. Entwicklungsfehler vor, da die besondere Gefährlichkeit der Autonomie zum Zeitpunkt des Inverkehrbringens ohne weiteres erkennbar war (lediglich der konkrete Schädigungsverlauf war unvorhersehbar).

Ein solcher Pflichtverstoß kann allerdings nur grundsätzlich bejaht werden und bleibt abhängig von den Umständen des Einzelfalls. Ist eine räumliche Begrenzung sichergestellt oder hat zumindest der Benutzer eine besondere Sachkunde, die ihm ein Eingreifen in autonome Verhaltensweisen des Roboters und damit eine Risikobeherrschung ermöglicht, wäre ein sorgfaltsgemäßes Inverkehrbringen durchaus denkbar. Bei einer intendier- 
ten Benutzung durch Verbraucher erscheint dies jedoch zumindest zum gegenwärtigen Zeitpunkt kaum möglich.

Durch die Abwägung im Einzelfall bleibt auch die Erprobung und Weiterentwicklung autonomer Roboter pflichtgemäß, sofern sie hinreichend abgesichert erfolgt. Dieser Ansatz ermöglicht eine verantwortungsvolle Technolgieentwicklung.

\section{Zulassung autonomer Roboter durch gesetzliche Regelung}

Will man, was eine politische Entscheidung ist, den Einsatz autonomer Maschinen außerhalb beherrschbarer Räume grundsätzlich ermöglichen, so wird man um eine rechtliche Klarstellung der entsprechenden Verhaltenspflichten nicht herumkommen. Ein entsprechendes Gesetz müsste regeln, dass bei Einhaltung des jeweiligen Stands der Informationstechnik oder Robotik (der wiederum durch technische Normung festgelegt wird) ${ }^{65}$ autonomes Verhalten nicht zu einem Produktfehler bzw. zu einem Produktsicherungspflichtverstoß führt.

\section{Einschränkung der Autonomie als Herstellerpflicht zumindest für eine Übergangszeit}

$\mathrm{Ob}$ ein Gesetz, das autonome Roboter ohne umfassende Einschränkungen zulässt, verfassungskonform und mit staatlichen Schutzpflichten vereinbar wäre, kann wegen der bestehenden Risiken bezweifelt werden. Als gesetzgeberische Alternative könnte auch das Inverkehrbringen autonomer Systeme zunächst ausdrücklich verboten werden (ergänzt um Erprobungsmöglichkeiten) und eine Pflicht der Hersteller normiert werden, Änderungen der Verhaltensregeln nur nach vorheriger Prüfung durch das Unternehmen zuzulassen (z.B. durch ein entsprechend geprüftes zentralisiertes Softwareupdate). Diese Vorgehensweise entspricht dem aktuell Üblichen.

65 Gruber, Bioinformationsrecht (Fn. 55), S. 291 f., weist jedoch zu recht darauf hin, dass bei autonomen Maschinen technische Normung eine geringere Wirkung hat bzw. weniger zuverlässig technische Sicherheit schaffen kann als bei herkömmlichen Maschinen. 


\section{Produktbeobachtung}

Soweit das Inverkehrbringen keine Haftung auslöst, bleibt der Verantwortungsbereich des Herstellers für die Beobachtung der in Verkehr gebrachten Roboter. Bei autonomen Geräten gewinnt die Produktbeobachtungspflicht besondere Bedeutung. Kommt es zum Erlernen gefährlicher Verhaltensweisen (,Anlernfehler"66), die dem Hersteller erkennbar sind, so hat er die notwendigen Maßnahmen (Information, Rückruf etc.) zu ergreifen. Tut er dies, so bleibt er, wenn der Vertrieb des autonomen Systems pflichtgemäß war, haftungsfrei.

\section{Einsatz autonomer Roboter}

\section{Einsatz als Verkehrspflichtverletzung}

Auch beim Einsatz autonomer Maschinen, deren besonderes Risiko bislang kaum beurteilt werden kann, muss man grundsätzlich von einer Sorgfaltspflichtwidrigkeit ausgehen. Im Einzelfall kann sich durch eine entsprechende Risikoverringerung (Einschluss oder kontinuierliche Überwachung des Roboters etc.) etwas anderes ergeben. Durch gesetzliche Regelung könnte konkretisiert werden, unter welchen Voraussetzungen (Größe, Art des Einsatzes etc.) ein Einsatz zulässig sein soll.

Wichtig erscheint es, die Erprobung der neuen Technologie zu ermöglichen. Nur so ist eine Weiterentwicklung und insbesondere auch eine Verbesserung der Sicherheit möglich. Dagegen erscheint das Bedürfnis für einen regulären Einsatz autonomer Roboter die ungewissen Risiken nicht aufzuwiegen.

\section{Geschäftsherrenhaftung sowie Roboter als Kinder}

Wie auch bei hoch- und vollautomatisierten Robotern wäre eine Analogie zu § 831 Abs. 1 BGB denkbar (vgl. C.IV.2). Autonome Roboter könnten in Unternehmen wie Verrichtungsgehilfen eingesetzt werden. Auch im privaten Bereich würde der Einsatz kostengünstigerer Roboter vermutlich wei-

66 A. Auer-Reinsdorff, persönlicher Vortrag am 28.10.2015, München. 
ter verbreitet sein als derjenige menschlicher Dienstleister zum jetzigen Zeitpunkt. Sofern der Einsatz autonomer Roboter jedoch gesetzlich zugelassen wurde, wird dem Anwender der Entlastungsbeweis gelingen.

Aus technischer Sicht könnte der Vergleich autonomer Roboter mit Kindern, die ebenfalls erst Verhaltensweisen erlernen müssen, zumindest erwägenswert sein. Daher könnte man an eine Analogie zu $\S 832$ Abs. 1 BGB denken. ${ }^{67}$ Auch hier handelt es sich aber wie bei $\S 831$ Abs. 1 BGB um eine Haftung für vermutetes Verschulden, so dass dem Betreiber der Entlastungsbeweis möglich ist. Ob auch das Anlernen eines autonomen Roboters derart im öffentlichen Interesse liegt, dass die Grundsätze des BGH für die Bestimmung der Aufsichtspflicht unter Berücksichtigung der gesetzlichen Wertung des $\S 1626$ Abs. 2 S. 1 BGB (die wachsende Fähigkeit und das wachsende Bedürfnis des Kindes zu selbständigem verantwortungsbewusstem Handeln sollen berücksichtigt werden) ${ }^{68}$ angewandt werden können, mag bezweifelt werden.

\section{Gefährdungshaftung in Analogie zur Tierhalterhaftung}

Unabhängig von der Frage, ob eine Verschuldenhaftung eingreift, könnte de lege lata auch eine Gefährdungshaftung bestehen. Zwar gibt es noch keine spezielle Gefährdungshaftung für Roboterrisiken (dazu sogleich), in Betracht kommt aber eine Analogie zur Tierhalterhaftung.

Da Autonomie eine Eigenschaft ist, die vor allem höheren Lebewesen zukommt, drängen sich Parallelen zwischen dem Einsatz von autonomen Robotern und von Lebewesen geradezu auf. Der naheliegendste Vergleich beim Einsatz von Robotern ist derjenige mit dem Halten von Tieren. ${ }^{69}$ Nach $\S 833$ BGB haftet der Halter eines Haustiers, das nicht dem Beruf, der Erwerbstätigkeit oder dem Unterhalt des Tierhalters zu dienen bestimmt ist, verschuldensunabhängig.

67 Vgl. A. Auer-Reinsdorff, persönlicher Vortrag am 28.10.2015, München; Günther, Roboter und rechtliche Verantwortung (Fn. 16), S. $135 \mathrm{ff}$.

68 BGH, Urt. v. 15.11.2012 - I ZR 74/12, NJW 2013, 1441, 1442 (Rn. 26) - Morpheus.

69 Vgl. Gruber, Zumutung und Zumutbarkeit (Fn. 24), S. 139; Günther, Roboter und rechtliche Verantwortung (Fn. 16), S. 138 ff.; A. Auer-Reinsdorff, persönlicher Vortrag am 28.10.2015, München. 
Die Vergleichbarkeit von Tieren und autonomen Robotern ergibt sich gerade aus der Unvorhersehbarkeit ihres Verhaltens. In Anlehnung an das spezifische Tierrisiko könnte man von einem spezifischen Roboterrisiko sprechen. Ratio legis des $\S 833$ BGB ist es, das besondere Risiko (hinsichtlich Eintrittshäufigkeit, möglicherweise auch Schadenshöhe und Beherrschbarkeit) ökonomisch dem Tierhalter aufzubürden. Dieser steht der Gefahrenquelle am nächsten und kann auf das Tier am besten einwirken.

Unabhängig von der Frage, ob ein Analogieschluss zu einer Gefährdungshaftungsregelung überhaupt zulässig sein soll oder ob die für eine Analogie sprechenden Argumente besser als Argumente zur Schaffung einer neuen gesetzlichen Regelung herangezogen werden (Überlegungen de lege referenda), bestehen aber Bedenken im Hinblick auf den Regelungszweck. Während bei der Tierhaltung durchaus auf die bessere Beherrschung der Gefahrenquelle Tier durch den Halter abgestellt werden kann, trifft dies auf denjenigen, der einen autonomen Roboter einsetzt, gerade nicht zu. Insbesondere, wenn es sich um einen Verbraucher handelt, dürfte er außer der Entscheidung, überhaupt einen Roboter einzusetzen, kaum Einfluss auf die Verwirklichung des Autonomierisikos haben. Dies hat nur der Hersteller, der durch eine entsprechende Programmierung versuchen kann, das autonome Verhalten bzw. die Herausbildung neuer Verhaltensweisen entsprechend zu steuern und zu begrenzen. Das Risiko autonomer Roboter ist als typisches Technikrisiko bzw. technisches Produktrisiko einzustufen, die in der Systematik der Gefährdungshaftungstatbestände ohnehin sehr problematische Tierhalterhaftung passt daher eher nicht.

\section{E. Roboterhaftung de lege ferenda}

Hoch- bzw. vollautomatisierte und autonome Roboter stellen eine neuartige Technologie dar, deren Risiken das Recht durch spezifische Haftungsregelungen zuweisen kann. Dabei handelt es sich um eine klassische Frage der Technikhaftung.

\section{Aufgaben des Haftungsrechts im Zusammenhang mit Risiken der Robotertechnologie}

Technikrecht, verstanden als techniksteuerndes Recht, kann Technik sowohl fördern (durch Entwicklungsanreize und Erleichterung des Techno- 
logietransfers) als auch begrenzen (insbesondere durch technisches Sicherheits- und Haftungsrecht). Während das Sicherheitsrecht die Grenzen des erlaubten Risikos festlegt, übernimmt das Haftungsrecht die Zuweisung der verbleibenden Risiken.

\section{Schadensprävention}

Die wichtigste Funktion des Haftungsrechts lässt sich ökonomisch verstehen: Haftungsrecht dient der Schadensvermeidung. ${ }^{70}$ Die Zuweisung der ökonomischen Risiken hat zugleich eine Steuerungsfunktion, die das Verhalten sowohl potenzieller Schädiger (Technikanwender) als auch potenzieller Geschädigter beeinflusst.

\section{a) Verschuldenshaftung}

Die Verschuldenshaftung setzt für den Technikanwender Anreize zu einer pflichtgemäßen Technikanwendung (Steuerung des Sorgfaltsniveaus). Dies setzt allerdings voraus, dass die Voraussetzungen der erlaubten Techniknutzung, also die konkreten Verkehrspflichten, sicher erkennbar sind. Bei einer richterrechtlichen Festsetzung von Verkehrspflichten kann dies problematisch sein. Das Ziel der Haftung für pflichtwidriges Verhalten sollte es aus ökonomischer Sicht sein, die Sorgfaltspflichten so einzustellen, dass sich ein optimaler gesellschaftlicher Gesamtnutzen ergibt. Dabei spielt neben der Möglichkeit einer Risikoverringerung durch den Handelnden auch die Möglichkeit einer Schadensabwehr durch die potenziell Geschädigten eine Rolle.

\section{b) Gefährdungshaftung}

Eine Gefährdungshaftung internalisiert Risiken vollständig, d.h. sie weist sie ökonomisch dem Verursacher zu, unabhängig davon, ob dieser bei der Risikosetzung rechtmäßig handelt. Dadurch wird dieser nicht nur dazu veranlasst, grösstmögliche Sorgfalt walten zu lassen (um einen Schaden

70 Kötz/Wagner (Fn. 32), Rn. 59. 
zu vermeiden), sondern er wird auch das Aktivitätsniveau so wählen, dass der erwartete Nutzen der Aktivität das Risiko überwiegt. Lohnt sich ein Risiko nicht, so wird die riskante Tätigkeit unterbleiben, solange sich nicht im Zuge der Weiterentwicklung ein verbessertes Nutzen-Risiko-Verhältnis ergibt. ${ }^{71}$ Die Risikobeurteilung nimmt dabei nicht der Staat vor, sondern der Technikanwender. Der Staat delegiert die Risikoabschätzung und erschließt so private Risikoinformation. ${ }^{72}$

Mit dieser Steuerungswirkung ist auch ein Anreiz für die Erforschung und Weiterentwicklung gegeben. ${ }^{73}$ Gerade im Hinblick auf neuartige Risiken sind Anreize für eine Weiterentwicklung bzw. Verbesserung besonders wichtig.

\section{Schadensstreuung}

Die Gefährdungshaftung kann eine Funktion der Schadensstreuung übernehmen wie sie auch der Produkthaftung zugrunde liegt. ${ }^{74}$ Allerdings tragen die Geschädigten zumindest das Insolvenzrisiko des Haftenden, was nur durch Sicherstellungspflichten (Kapitalausstattung, Haftpflichtversicherung) verhindert werden kann.

Durch die Verteilung des Risikos auf die Schultern aller Techniknutzer kann eine entsprechende Haftungsregelung mit Versicherungspflicht die Einführung einer riskanten Technologie unter Umständen überhaupt erst ermöglichen. Als Alternative kommt hier aber auch eine Unfallversicherung in Betracht (dazu unter IV.).

\section{Technikermöglichung}

Ganz allgemein kann Gefährdungshaftungsregelungen, die neuartige Technologien betreffen, die Funktion der Technikermöglichung zugespro-

71 Kötz/Wagner (Fn. 32), Rn. 506 ff.; Zech (Fn. 29), S. 21 (24) (jeweils m.w.N.).

72 G. Wagner, Haftung und Versicherung als Instrumente der Techniksteuerung, VersR 1999, S. 1441 (1444).

73 Wagner, Haftung und Versicherung als Instrumente der Techniksteuerung, VersR 1999, S. 1441 (1444); A. Röthel, Zuweisung von Innovationsverantwortung durch Haftungsregeln, in: M. Eifert/W. Hoffmann-Riem (Hrsg.), Innovationsverantwortung, Berlin 2009, S. 335 (349); Zech (Fn. 29), S. 21 (25).

74 Kötz/Wagner (Fn. 32), Rn. $90 \mathrm{ff}$. 
chen werden. Gefährdungshaftungsregelungen werden dann eingeführt, wenn besonders hohe Risiken (Schadenshöhe, Eintrittswahrscheinlichkeit) oder schlecht abwehrbare Risiken auftreten, die gleichwohl wegen ihres potenziellen Nutzens nicht einem Verbot unterworfen werden sollen. Dabei ist die Ungewissheit bei der Beurteilung kein Hinderungsgrund, sondern kann sogar ein Grund für die Einführung einer Gefährdungshaftung darstellen (wie etwa bei der Gentechnik). Wichtig ist, dass Gefährdungshaftungsregeln - anders als sicherheitsrechtliche Verbote oder einschränkende Verkehrspflichten - von der grundsätzlichen Erlaubtheit des riskanten Handelns, insbesondere der Techniknutzung ausgehen, sie stellen „freiheitsbetonende Unsicherheitenregel[n]“ dar. ${ }^{75}$ Der unbedingte Schadensausgleich kann Technikakzeptanz steigern und auch dadurch Technikanwendung ermöglichen.

\section{Spezifische Risiken der Robotertechnik und tatbestandliche Erfassung}

Als spezifische Risiken der Robotertechnik, die tatbestandlich erfasst werden könnten, kommen Automatisierungs- und Autonomierisiken in Betracht. Haftungsregelungen könnten nur autonome Roboter oder auch hoch- und vollautomatisierte Maschinen erfassen.

Sie könnten zudem nach den Kriterien abgrenzen, die bereits unter C.I und D.I besprochen wurde, also Automatisierungs- bzw. Autonomiegrad, Größe und Energiefreisetzung, Mobilität oder räumliche Eingrenzung, gewerblicher oder privater Einsatz und. Qualifikation oder Zulassungsvoraussetzungen für den Einsatz.

75 I. Spiecker gen. Döhmann, Rechtliche Begleitung der Technikentwicklung im Bereich moderner Infrastrukturen und Informationstechnologien, in: H. Hill/U. Schliesky (Hrsg.), Die Vermessung des virtuellen Raums, Baden-Baden 2012, S. 137 (152 f.). Vgl. ebd., S. 153: „Daher sind Haftungsregeln Ausdruck einer Unsicherheitenregel, die zwar einen grundsätzlichen Ausgleich der Positionen von Risiko und Chance wahrt, aber in der Tendenz der positiven Bewertung den Vorrang einräumt."; M. Rohe, Gründe und Grenzen deliktischer Haftung, AcP 201 (2001), S. 117 (138): „Schaffung besonderer, aus übergeordneten Gründen erlaubter Risikopotentiale". 


\section{Einführung einer besonderen Roboter-Produkthaftung (Gefährdungshaftung der Hersteller)}

Stellt man das Inverkehrbringen autonomer Systeme frei, d.h. wird dieses durch gesetzliche Regelung als pflichtkonform statuiert, so kommt für eine effiziente Risikoverteilung die Einführung einer Gefährdungshaftung des Herstellers in Betracht. ${ }^{76}$

Als Vorbild einer solchen Haftung wäre die Haftung für gentechnische Produkte nach $\S 37$ II GenTG zu nennen, die auch Entwicklungsrisiken umfasst. Anders als die normale Produkthaftung handelt es sich hierbei nicht um eine Haftung für pflichtwidriges Verhalten, sondern um eine genuine Gefährdungshaftung für die Nutzung einer besonderen Technologie durch den Hersteller entsprechender Produkte.

$\mathrm{Zu}$ einem ähnlichen Ergebnis kommt man, wenn man eine eigene Gefährdungshaftung für die wirtschaftliche Nutzung technischer Neuerungen fordert. ${ }^{77}$ Wirtschaftlicher Nutzer wäre dann in erster Linie derjenige, der Roboter vertreibt, unter Umständen auch unternehmerische Betreiber, nicht jedoch private Betreiber.

Für eine solche Technikhaftung bzw. eine Gefährdungshaftung des Herstellers spricht vor allem der Anreiz zur Weiterentwicklung der Technologie. Zwar ist der Hersteller nicht notwendig mit dem Entwickler identisch. Jedoch ist er in der Regel mit diesem durch Lizenzverträge verbunden, da es sich um neuartige und damit schützbare (sei es durch Schutz des Unternehmensgeheimnisses, sei es durch Patentrecht) Technologien handelt. Allerdings könnte sie auch dazu führen, dass eine gesellschaftlich erwünschte Einführung von Robotertechnologie zunächst unterbleibt, da die Risiken für die Hersteller nicht kalkulierbar sind.

76 Befürwortend Lohmann, EJRR 2/2016 (Fn. 54), S. 335 (337). Vgl. Hanisch (Fn. 36), S. 27 (54 ff.).

77 Röthel, (Fn. 73), S. 351 ff.; Zech (Fn. 29), S. 21 (27f.); ablehnend Ch. Ann, Die Haftung für Schäden aus technologischer Innovation - Zur Tauglichkeit des Haftungsrechts als Instrument für ein volkswirtschaftlich sinnvolles Risikomanagement, in: Seehafer u.a. (Hrsg.), Risikoregulierung und Privatrecht, Stuttgart 1994, S. 54; J. Eichelberger, Innovationsrelevante Regeln des allgemeinen Zivilrechts und ihre Innovationswirkung, in: R. M. Hilty/Th. Jaeger/M. Lamping (Hrsg.), Herausforderung Innovation, Berlin/Heidelberg 2012, S. 53 f. 


\section{Einführung einer Roboter-Verkehrshaftung (Gefährdungshaftung der Betreiber)}

Es stellt sich die Frage, ob nach dem Vorbild bestehender Technikhaftungstatbestände eine eigene Haftung für den Einsatz von Robotern geschaffen werden sollte. ${ }^{78}$ Als Vorbild bietet sich hier insbesondere die Halterhaftung bei Kraftfahrzeugen an. Auch hier handelt es sich um eine Technologie, deren Einsatz gesellschaftlich gewünscht, jedoch mit hohen Risiken (insbesondere Schadenshäufigkeit, aber auch Schadenshöhe) verbunden ist. Die Lösung besteht darin, den Einsatz unter entsprechenden Voraussetzungen (Zulassungspflicht) zuzulassen, jedoch mit einer Gefährdungshaftung zu verbinden, die durch eine Pflicht zum Abschluss einer Haftpflichtversicherung sichergestellt wird. Es handelt sich um eine klassische Gefährdungshaftung zur Ermöglichung von Techniknutzung. Eine entsprechende Lösung dürfte sich auch für Roboter anbieten. In der Tat wird voraussichtlich der Straßenverkehr mit selbststeuernden Fahrzeugen der erste Anwendungsfall einer entsprechenden gesetzlichen Regelung sein. Hier gibt es bereits konkrete Pläne, neben der ohnehin bestehenden Gefährdungshaftung klarzustellen, dass die Nutzung eines zugelassenen selbststeuernden Autos ohne eigenes Eingreifen keinen Pflichtverstoß darstellt. ${ }^{79}$

Ein Bedenken gegen eine Haftung der Anwender liegt darin, dass sie, anders als eine Haftung der Hersteller, keine Anreize für eine Weiterentwicklung von Technologien setzt. Dies ist besonders dann wichtig, wenn es sich um neuartige Technologien handelt, deren Risiken durch weitere Erforschung und Entwicklung verringert werden können. Anders als herkömmliche Straßenverkehrstechnik, die einen hohen Grad an technologischer Reife aufweist, lässt sich die Roboter-Technik, insbesondere bei autonomen Robotern noch lange nicht als ausgereift bezeichnen.

78 Dazu Gruber, Zumutung und Zumutbarkeit (Fn. 24), S. 145 ff.; ders., Bioinformationsrecht (Fn. 55), S. 279 ff.; Hanisch (Fn. 36), S. 27 (46 ff.); Horner/Kaulartz (Fn. 24), S. 7 (13 f.); vgl. Christaller u.a. (Fn. 3), S. 154 ff.; John, (Fn. 60), S. 276 ff. (lehnt Analogieschluss zu bestehenden Gefährdungshaftungsregelungen

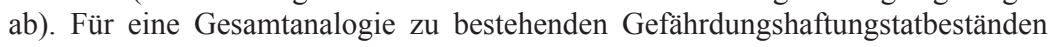
Kötz/Wagner (Fn. 32), Rn. 514.

79 FAZ vom 11.7.2016, S. 19, Ethikkommission für Roboterautos: „,[Bundesverkehrsminister] Dobrindt zufolge soll sichergestellt werden, dass Autofahrer bei ordnungsgemäßer Nutzung des Autopiloten ihre Sorgfaltspflicht nicht verletzen und ihnen so keine zusätzlichen Haftungsrisiken entstehen können.“ 
Allerdings lassen sich entsprechende Entwicklungsanreize auch anders setzen. Marktmechanismen können, wie bei anderen Produkten auch, dazu führen, dass über die Nachfrage für sichere Roboter genügende Anreize bestehen. Eine Gefährdungshaftung der Betreiber bzw. Halter würde dazu führen, dass Anreize zur Risikovermeidung beim Betrieb bestehen. Auch durch die notwendige Pflichtversicherung werden diese Anreize nicht zunichte gemacht, da sie über die Höhe der Versicherungsprämie erhalten werden können. Da sich das Prinzip bei Kraftfahrzeugen bewährt hat und durch die bestehenden Regelungen bei selbstfahrenden Fahrzeugen ohnehin zur Anwendung kommen wird, spricht vieles dafür, Roboterrisiken durch eine Betreiberhaftung mit Versicherungspflicht aufzufangen.

\section{Haftungsersetzende Unfallversicherung als Alternative zu Gefährdungshaftungsregelungen}

Als dritte Gestaltungsalternative ließe sich auch eine weitgehende Haftungsfreistellung sowohl der Hersteller als auch der Anwender denken, verbunden mit einer besonderen staatlichen Unfallversicherung, die die Risiken der Robotertechnologie sozialisiert. ${ }^{80}$ Sie erscheint aber den beiden Haftungsmodellen unterlegen, da sie weder Anreize für eine Weiterentwicklung der Robotertechnologie im Hinblick auf ihre Sicherheit setzt, noch eine Steuerungswirkung zur Risikovermeidung bei ihrem Einsatz entfaltet. Auch hier gibt es aber Möglichkeiten, Steuerungswirkung zu erhalten, insbesondere durch Rückgriffsansprüche im Wege der Legalzession (vgl. § 116 Abs. 1 SGB X).

\section{Haftung der Roboter selbst?}

Nur kurz angedeutet werden soll, welche Fragen zu klären sind, wenn man die Roboter selbst zu Haftungssubjekten machen möchte. Sollten Roboter dereinst wirklich Persönlichkeit entwickeln, wäre eine mögliche Konsequenz ihre Anerkennung als natürliche Rechtsperson. Allerdings ist die

80 Ann (Fn. 77), S. 55; Rohe (Fn. 75), S. 117 (133); Kötz/Wagner (Fn. 32), Rn. 9 (571 ff.). Zu den Vorzügen bei multifaktorieller Verursachung (wie sie durch die Vernetzung von Robotern häufig vorkommen wird) G. Wagner, Asbestschäden Bismarck was right, ZEuP 2007, S. 1122 (1132 ff.). 
Rechtsordnung diesen Weg auch für Tiere, die Selbstbewusstsein aufweisen, bisher nicht gegangen.

Eine gänzlich andere Frage ist es, ob eine juristische Person geschaffen werden soll, die als Haftungssubjekt für durch einen Roboter verursachte Schäden dient. ${ }^{81}$ Diese wäre nicht mit dem Roboter identisch. Zwar könnte der Roboter für eine solche juristische Person handeln. Dies wäre jedoch nur mit dem Einsatz von Maschinen zur Abgabe von Willenserklärungen zu vergleichen. Eine juristische Person muss von einem Organ vertreten werden, welches wiederum nur eine geschäftsfähige Person (meist auch nur eine natürliche Person, z.B. § 6 II 1 GmbHG) sein kann. Davon unabhängig bestehen weitere Voraussetzungen für die Schaffung juristischer Personen, insbesondere Publizitätserfordernisse und - wenn auch eingeschränkt - Kapitalerfordernisse. Diese gesellschaftsrechtlichen Fragen können hier nicht im Einzelnen erörtert werden. Es erscheint aber auch nicht praktikabel, den Betrieb von Robotern, die gerade als massenhafte Helfer im Alltag dienen sollen, an die Errichtung einer Betreibergesellschaft zu knüpfen. Dies wäre für private Anwender kaum zu leisten.

\section{F. Fazit}

Im Ergebnis besteht momentan - sieht man einmal von dem Sonderfall selbststeuernder Fahrzeuge ab - keine verschuldensunabhängige Haftung für den Einsatz von Robotern. Bei der Verschuldenshaftung besteht Klarstellungsbedarf, welche Arten des Einsatzes von Robotern pflichtgemäß sind und welche pflichtwidrig. Als Gretchenfrage für die Zukunft wird zu klären sein, ob der Einsatz autonomer Roboter außerhalb geschützter Räume zugelassen werden soll. Für die Übergangszeit, bis die Risiken autonomer Roboter hinreichend abschätzbar erscheinen, bietet es sich an, Hersteller zu verpflichten, Verhaltensänderungen nur per von Menschen geprüften Updates zuzulassen.

Um den flächendeckenden Einsatz von Robotern zu ermöglichen, bietet es sich zudem an, die besonderen Roboterrisiken durch eine besondere

81 Vgl. Hanisch (Fn. 36), S. 27 (39 f.); S. Beck, Technisierung des Menschen - Vermenschlichung der Technik, in: M.-Ch. Gruber/J. Bung/S. Ziemann (Hrsg.), Autonome Automaten - Künstliche Körper und artifizielle Agenten in der technisierten Gesellschaft, 2. Aufl., Berlin 2015, S. 173 (179); Lohmann, Automatisierte Fahrzeuge (Fn. 14), S. 393 f. 
Haftungs- oder Versicherungsregelung umzuverteilen. Dabei kommen wie allgemein bei neuartigen technischen Produkten - drei grundsätzliche Möglichkeiten in Betracht: eine Gefährdungshaftung der Hersteller, eine Gefährdungshaftung der Anwender mit Versicherungspflicht oder eine haftungsersetzende Unfallversicherung. 